OPEN ACCESS

Edited by:

Basil J. Nikolau,

lowa State University, United States

Reviewed by:

Shuangxia Jin

Huazhong Agricultural University,

China

Yonggen Lou,

Zhejiang University, China

*Correspondence:

Christian Hettenhausen hettenhausen@mail.kib.ac.cn

Jianqiang Wu

wujianqiang@mail.kib.ac.cn

Specialty section

This article was submitted to Plant Metabolism and Chemodiversity, a section of the journal

Frontiers in Plant Science

Received: 17 April 2017 Accepted: 22 September 2017 Published: 10 October 2017

Citation:

Song J, Liu H, Zhuang H, Zhao C, Xu Y, Wu S, Qi J, Li J, Hettenhausen C and Wu J (2017) Transcriptomics and Alternative Splicing Analyses Reveal

Large Differences between Maize Lines B73 and Mo17 in Response to Aphid Rhopalosiphum padi Infestation. Front. Plant Sci. 8:1738. doi: 10.3389/fpls.2017.01738

\section{Transcriptomics and Alternative Splicing Analyses Reveal Large Differences between Maize Lines B73 and Mo17 in Response to Aphid Rhopalosiphum padi Infestation}

\author{
Juan Song ${ }^{1,2}$, Hui Liu ${ }^{1}$, Huifu Zhuang ${ }^{1,2}$, Chunxia Zhao ${ }^{3}$, Yuxing $X u^{1,2}$, Shibo $W u^{4}$, \\ Jinfeng $Q i^{1}$, Jing $L^{1}{ }^{1}$, Christian Hettenhausen ${ }^{1 *}$ and Jianqiang $W u^{1 *}$ \\ ${ }^{1}$ Yunnan Key Laboratory for Wild Plant Resources, Department of Economic Plants and Biotechnology, Kunming Institute of \\ Botany, Chinese Academy of Sciences, Kunming, China, ${ }^{2}$ University of the Chinese Academy of Sciences, Beijing, China, \\ ${ }^{3}$ Dalian Institute of Chemical Physics, Chinese Academy of Science, Dalian, China, ${ }^{4}$ Yunnan Academy of Science and \\ Technology Development, Kunming, China
}

Maize (Zea mays L.) is a staple crop worldwide with extensive genetic variations. Various insects attack maize plants causing large yield loss. Here, we investigated the responses of maize B73, a susceptible line, and Mo17, a resistant line, to the aphid Rhopalosiphum padi on metabolite and transcriptome levels. R. padi feeding had no effect on the levels of the defensive metabolites benzoxazinoids (Bxs) in either line, and Mo17 contained substantially greater levels of Bxs than did B73. Profiling of the differentially expressed genes revealed that $\mathrm{B} 73$ and Mo17 responded to $R$. padi infestation specifically, and importantly, these two lines showed large gene expression differences even without R. padi herbivory. Correlation analysis identified four transcription factors (TFs) that might account for the high Bx levels in Mo17. Similarly, genome-wide alternative splicing (AS) analyses indicated that both B73 and Mo17 had temporally specific responses to $R$. padi infestation, and these two lines also exhibited large differences of AS regulation under normal condition, and 340 genes, including 10 TFs, were constantly differentially spliced. This study provides large-scale resource datasets for further studies on the mechanisms underlying maize-aphid interactions, and highlights the phenotypic divergence in defense against aphids among maize varieties.

Keywords: maize (Zea mays L.), Rhopalosiphum padi, transcriptome, alternative splicing, metabolites, benzoxazinoid

\section{SIGNIFICANCE STATEMENT}

This is a comprehensive study on the defense responses of maize lines B73 and Mo17 to aphid herbivory. Aphid Rhopalosiphum padi infestation-induced defense-related metabolites and genome-wide changes of gene expressions and alternative splicing (AS) patterns were investigated, and we show that different maize lines have distinct defense-related responses to $R$. padi herbivory. This large-scale comparison between B73 and Mo17 provides key resource information and candidate genes for further identification of the regulatory network underlying maize defense against aphids. 


\section{INTRODUCTION}

Aphids (Hemiptera: Aphididae) are the most common phloemfeeding pests worldwide. They are devastating to host plants, including crops, due to their capability of very rapid population growth, deprivation of host photosynthates (carbon) and amino acids (nitrogen), reducing photosynthesis, and transmitting viruses (Hales et al., 1997; Smith and Boyko, 2007). The symptoms of aphid-attacked plants are diverse, such as chlorosis, necrosis, wilting, stunted growth, leaf curling, and gall formation (Guerrieri and Digilio, 2008), and vary according to aphid and plant species (Cooper and Goggin, 2005; Goggin, 2007).

Maize (Zea mays) is one of the most important crops as it is food to a large portion of the world population and is also used for animal feed and industry (Hu and $\mathrm{Xu}, 2011$; Waisi et al., 2015). Globally, plant pests cause about $20-40 \%$ annual crop production losses, including maize (Oerke, 2005; FAO, 2017). However, how maize responds to insect herbivory, including aphids, is still not fully understood. Benzoxazinoids (Bxs), also known as cyclic hydroxamic acids, are important direct defense metabolites in certain poaceous plants and were first reported in maize and wheat (Triticum aestivum L.) (Wahlroos and Virtanen, 1959). Using maize plants having a mutation in the Bx biosynthesis gene BENZOXAZINELESS1 (BX1), Ahmad et al. (2011) demonstrated that Bxs are very important metabolites in maize defense against $R$. maidis. Later, a genetic study further pointed to the toxicity of HDMBOA-Glc (2-hydroxy-4,7-dimethoxy-1,4-benzoxazin-3one glucoside) to $R$. maidis and the callose inducibility of DIMBOA-Glc (2,4-dihydroxy-7-methoxy-1,4-benzoxazin-3-one glucoside) (Meihls et al., 2013).

In addition to compounds that are repellent, anti-nutritive, or toxic to insects, which are named direct defenses (Glauser et al., 2011; Handrick et al., 2016), plants also produce volatiles after herbivory, and some of these are important foraging cues for natural enemies of herbivores. These volatiles function as plant indirect defenses (Heil, 2008; de Vos and Jander, 2010). For example, during beet army worm (Spodoptera exigua) feeding, maize seedlings can perceive the elicitor volicitin in the caterpillar oral secretions and activate the biosynthesis of terpene volatiles, which attract herbivore parasitoids (e.g., Cotesia marginiventris) (Alborn et al., 1997; Schnee et al., 2006). Similarly, Spodoptera littoralis-attacked maize seedlings emit mono- and sesquiterpenes, which help parasitic wasps locate the larvae (Schnee et al., 2002). Insect oviposition can also elicit terpene emission in maize and the parasitic wasps are attracted to the oviposited maize (Tamiru et al., 2011, 2017).

Transcriptomic analyses have revealed changes in the expression levels of various genes in aphid-attacked plants (Thompson and Goggin, 2006). For example, Appel et al. (2014) demonstrated that feeding of green peach aphid Myzus persicae and cabbage aphid Brevicoryne brassicae up- and downregulated various genes in Arabidopsis, but these genes were only $4-8 \%$ overlapping. In response to the corn leaf aphid (Rhopalosiphum maidis) infestation, maize line B73 showed dynamic transcriptomic changes, and the expression levels of hundreds of genes were altered at different time points investigated, with most of the genes up-regulated; further functional analyses revealed that most of the up-regulated genes were defense-related, while a number of down-regulated transcripts were related to primary metabolism (Tzin et al., 2015a). Another microarray study revealed that M. persicae infestation on Arabidopsis resulted in the largest number of changes in gene expressions, compared with those induced by pathogens Pseudomonas syringae and Alternaria brassicicola, chewing caterpillar Pieris rapae, and cell content-feeding thrips Frankliniella occidentalis (De Vos et al., 2005).

Alternative splicing (AS) is an important post-transcriptional regulatory mechanism in eukaryotes that processes premature mRNA into multiple mature isoforms, thus affecting mRNA stability and increasing transcriptome complexity and protein diversity (Black, 2003; Simpson et al., 2008). AS is involved in plant growth and development (Romanowski and Yanovsky, 2015; Srinivasan et al., 2016; Wang et al., 2017) and adaptation to abiotic and biotic stresses (Schliebner et al., 2014; Ren et al., 2015; Keller et al., 2016; Thatcher et al., 2016; Jiang et al., 2017). Recently, in response to the lepidopteran chewing insect Manduca sexta, 180 and 356 differentially spliced genes were identified in the roots and leaves of the wild tobacco Nicotiana attenuata, respectively, suggesting a role of AS in regulating plant defense against lepidopteran insects (Ling et al., 2015). However, whether and how AS is involved in plant resistance to aphids was unknown.

Many studies have revealed very large genetic diversity in maize (Buckler et al., 2006), leading to broad variations in its resistance to insect herbivores (Meihls et al., 2012; Tzin et al., 2015b). For example, $R$. maidis produced about 20 times more progeny on the inbred line B73 than on Mo17 (Betsiashvili et al., 2015), and six commonly grown maize hybrids in Iran, namely K3640/3 × MO17, Simon, SC704, EXP1, VRE26 $\times$ K18, and VRE27 $\times \mathrm{K} 18$, showed large differences in resistance to R. maidis (Razmjou and Golizadeh, 2010). In this study, we used mass spectrometry-based metabolite and Illumina RNA-seq analyses to investigate the defense responses to $R$. padi, a commonly occurring insect pest in maize, in two different maize inbred lines, the resistant line Mo17 and the susceptible line B73. Our data reveal extensive differences between B73 and Mo17 in their responses to $R$. padi feeding on metabolite and transcriptome levels, including gene expression and AS patterns. These data provide new insight into the regulatory elements that might shape the differences between B73 and Mo17.

\section{MATERIALS AND METHODS}

\section{Plant Growth and Aphid Rearing}

Seeds of maize inbred lines B73, Zong3, Chang7-2, A188, and Mo17 were sown in $12 \mathrm{~cm}$-diameter plastic pots filled with humus soil and vermiculite (about 7 to 1 ratio). Plants were grown in a greenhouse at $\sim 17^{\circ} \mathrm{C}$ (night) and $\sim 28^{\circ} \mathrm{C}$ (day) under a $14 \mathrm{~h}$ photoperiod until the fourth leaf had fully expanded (V4, 3-weekold seedlings). A R. padi colony was established from a single first instar nymph collected from a field in the Kunming Institute of Botany, Chinese Academy of Sciences, in Kunming, China, in 2014. The insect colony was maintained in an $80 \times 80 \times 150 \mathrm{~cm}$ cage on sorghum plants at $17-28^{\circ} \mathrm{C}, 14 \mathrm{~h}$ day length. 


\section{Aphid Bioassays}

To evaluate the survival rates of $R$. padi, 20 apterous adults were transferred to the middle of the forth leaves and enclosed in clip cages ( $3 \mathrm{~cm}$ diameter), and after $24 \mathrm{~h}$, the adult aphids were brushed off and 20 neonates were left in the clip cages to evaluate every day in the next 5 days (20 replicated maize plants for each group) (Carena and Glogoza, 2004; Mao and Zeng, 2014). To determine days from birth to first reproduction, life span, and progeny per aphid, one new neonate was placed on the middle of the forth leaf of each plant and the performance parameters were estimated until its death (20 replicated maize plants for each group) (Kettles et al., 2013).

\section{Plant Treatments and Sample Collection}

For treatment on maize, aphid feeding and mock treatments were performed as the following: for each aphid-treated plant, 50 adult aphids were gently placed onto the middle of the fourth leaf and enclosed in a clip cage, and the plants of the control group (mock) were fixed with empty clip cages. Three replicates were used for the aphid feeding and control group. In order to minimize the variations caused by circadian rhythm and other differences in environmental conditions, we treated leaves at different time points prior to the collection time. Aphids were quickly removed with a soft paintbrush at the time of harvest, and the regions in the cages were immediately excised and snap-frozen in liquid nitrogen.

\section{Metabolite Data Acquisition and Analyses}

50 adult aphids were feeding on plant for 0 and $48 \mathrm{~h}$, five biological replicates were used, and a previously established method was employed (Qi et al., 2016). In brief, samples were extracted with $80 \%$ methanol and then analyzed on an Agilent 1200 Rapid Resolution Liquid Chromatography (RRLC) system equipped with a ZORBAX SB-Aq column $(2.1 \times 100 \mathrm{~mm}$, $1.8 \mu \mathrm{m}$ ) followed by an Agilent $6510 \mathrm{Q}-\mathrm{TOF}$ running in positive ionization mode. The column temperature was set at


were identified based on pure standards, except some Bxs: DIMBOA was confirmed by comparing the Bx profiles between maize inbred line $\mathrm{H} 88$ and $b x 1$ mutant (GRMZM2G085381); DIMBOA-Glc, DIM 2 BOA-Glc, HDMBOA-Glc, and MBOA were confirmed with purified standards, and the other putative Bxs were identified by their molecular masses. The abundance of each metabolite was estimated from the peak area. Peak detection and matching were performed using bioconductor XCMS and CAMERA packages (http://www.bioconductor.org/.) Samples (metabolites after integration) with $P<0.05$ were considered to be differentially regulated. The acquired data were introduced to the SIMCA-P software package (v11.5, Umetric, Umea, Sweden) for Principal Component Analysis (PCA). The data was filtered by orthogonal signal correction (OSC) to remove variations from non-correlated factors.

\section{RNA-Seq Data Acquisition and Analysis of Differentially Expressed Genes}

Three biological replicates were used for RNA-seq analyses. Total RNA was extracted from ground leaf samples using
TRIzol reagent (Invitrogen). The concentration of total RNA was measured using a NanoDrop 2000 Spectrophotometer (Thermo Scientific). Sequencing was performed at $3.75 \mathrm{G}$ depth on a HiSeq2500-PE125 platform (Illumina). The raw sequence data are deposited in NCBI's Short Read Archive under the BioProject ID (PRJNA338511). The resulting sequences were trimmed based on quality scores and mapped to the maize B73 reference genome sequence V2 and maize working gene set V5a with Tophat2 (Kim et al., 2013) using the following modifications from the default parameters: maximum intron size 100,000; minimum intron size 20; up to two mismatches allowed (Trapnell et al., 2012). The expression levels of genes were estimated using Cufflink and were normalized using the numbers of reads per $\mathrm{kb}$ of exon sequence in a gene per million mapped reads. Differential expression analysis was performed using the Cuffdiff (Trapnell et al., 2012) with a cutoff of 2 -fold change relative to the control levels.

\section{Alternative Splicing Analysis}

All AS analyses were based on splice junctions obtained from the BAM files produced by TOPHAT2. For differential splicing analysis, we used Cuffdiff to categorize individual transcripts based on their transcription start site (Sammeth et al., 2008), thus grouping all the transcripts with a common premRNA molecule. Thereafter, we performed Cuffdiff to estimate significant expression differences of each transcript using the Jensen-Shannon divergence metric across the different samples (Trapnell et al., 2012).

\section{Functional Annotation and Classification}

For gene ontology (GO) analysis, we obtained the GO terms for each maize gene using the AgriGO web service (Du et al., 2010), and WEGO (Ye et al., 2006) was used for GO functional annotation; Plant MetGenMAP (Joung et al., 2009) was used for pathway enrichment analysis. Sets of genes showing a similar pattern of transcription were assumed to be functionally correlated. Transcription patterns were clustered using $\mathrm{MeV}$ 4.9.0 (Saeed et al., 2003) with Euclidean distances and the hierarchical cluster method of complete linkage clustering and Java software. MeV 4.9.0 was also used to create heat-map visualizations of differentially expressed genes. To identify the transcription factors, we referenced a maize transcription factor database retrieved from the Plant Transcription Factor Database 4.0 (Jin et al., 2015, 2017).

\section{RESULTS}

\section{Determination of the Resistance Levels of Different Maize Lines to $R$. padi}

To evaluate the levels of resistance to $R$. padi in different maize inbreed lines, we measured the survival rates of $R$. padi on the fourth fully expanded leaves of the inbred lines B73, Zong3, Chang7-2, A188, and Mo17 for 6 days (Figure 1A). R. padi showed the highest survival rate on B73 (86.3\% survived on day 1), while exhibited the lowest survival rate on Mo17 (69\% survived on day 1). The survival rates decreased in B73 and Mo17 and the trend continued in the next 5 days (65.6 and 10.5\% aphids survived on B73 and Mo17 respectively on day 6) (Figure 1A). 
Congruently, the life spans of $R$. padi on B73 and Mo17 were 22.9 and 18.6 days, respectively, and the new neonates on B73 needed 8.7 days to start to give offspring, while 11 days were needed on Mo17 (Figure 1B). R. padi fecundity on B73 was much higher than on Mo17 (39.7 and 12.9 offspring per aphid on B73 and Mo17, respectively) (Figure 1C). Thus, B73 and Mo17 are a $R$. padi-susceptible and a-resistant line, respectively. These results were similar to previous findings, where Mo17 exhibited a much greater defense level against the maize leaf aphid $R$. maidis than did B73 (Meihls et al., 2013; Betsiashvili et al., 2015).

\section{Changes of Metabolites after R. padi Feeding}

To explore the metabolites in B73 and Mo17 that might account for the observed $R$. padi resistance, leaf samples were collected $48 \mathrm{~h}$ after $R$. padi infestation. The profiles of $\mathrm{Bx}$ and phenolic metabolites were obtained on an UPLC-Q-TOF MS system (Table S1).

Given the importance of Bxs in maize defense against insects (Long et al., 1977; Rostas, 2007; Niemeyer, 2009; Glauser et al., 2011), we quantified the contents of Bxs (Figure 2A, Table S1). In general, Bxs were much higher in Mo17 than in B73. For example, DIMBOA, DIM 2 BOA, DIMBOA-Glc, and DIM ${ }_{2}$ BOA-Glc in Mo17 were at least 2-fold > B73 (Figure 2A, Table S1). Notably, $R$. padi feeding did not have an obvious impact on the contents of Bxs in either line. Another group of metabolites that we examined were the phenolics. The contents of phenolics also showed large differences between B73 and Mo17. Without R. padi treatment, 10 phenolic compounds, apigenin O-hexoside, apigenin C-hexoside C-pentoside, quercetin O-hexoside, quercetin O-deoxyhexosyl-hexoside, luteolin, luteolin O-hexoside, luteolin O-hexoside O-deoxyhexoside, neochlorogenic acid, isorhamnetin O-hexoside, and isorhamnetin O-rhamnoglycoside were 0.64-3.29-fold higher in B73 than in Mo17, while the content of scytalone was $34.9 \%$ lower in B73 (Figure 2B, Table $\mathrm{S} 1)$. After $48 \mathrm{~h}$ of $R$. padi feeding, most phenolics decreased in B73 (39-77\%), but remained constant in Mo17.

\section{R. padi Infestation-Induced Regulation of Gene Expressions in B73 and Mo17}

We used RNA-seq technology to analyze the genome-wide gene expression responses of $\mathrm{B} 73$ and Mo17 to R. padi feeding at 6 and $24 \mathrm{~h}$.

Differentially expressed genes (DEGs) were selected based on significance $(P<0.05)$ (Table S2): 3,789 and 3,420 DEGs were found in B73 after 6 and $24 \mathrm{~h} R$. padi feeding, and the same treatment resulted in 4,127 and 1,786 DEGs in Mo17. Gene functions were further classified using GO analysis. As shown in Figure S1 (Table S2): 5,657 DEGs in B73 and 4,683 DEGs in Mo17 were assigned to $25 \mathrm{GO}$ terms (Cellular Component, Molecular Function, and Biological Process: 8, 6, and 11 GO terms, respectively). The GO terms in B73 and Mo17 were largely similar except that some from Cellular Component (Extracellular Region Part and Macromolecular Complex) and Biological Process (such as Biological adhesion and Cellular Component Organization) showed significant differences.
Detailed expression profiling, based on the criteria that the genes should be up- or down-regulated more than 1-fold and $P<0.05$, indicated that both $\mathrm{B} 73$ and Mo17 responded to $R$. padi infestation, but in a different manner (Table S3). After $6 \mathrm{~h}$ of R. padi feeding (Figure 3A, Table S3), 745 (319 up- and 426 down-regulated) and 853 (555 up- and 298 down-regulated) genes showed changes in expression levels in B73 and Mo17, respectively, and among these, only 162 were regulated in both lines. After $24 \mathrm{~h}$ of $R$. padi infestation (Figure 3B, Table S3), still 567 DEGs were uniquely regulated in B73, while Mo17 had specific 221 DEGs; only 80 genes showed altered levels in both B73 and Mo17 at $24 \mathrm{~h}$. Thus, B73 and Mo17 have very different transcriptomic responses to $R$. padi infestation.

The DEGs were screened with a cluster analysis based on the k-means method using Pearson's correlation distance (TM4 software, http://www.tm4.org/). Each cluster represents genes whose expressions showed similar response patterns to $R$. padi infestation (Figure 4A, Table S4). In B73, 1,157 genes were classified into three expression cluster groups (Clusters 1-3). B73 Cluster 1, which contained 338 genes, showed a positive slope from 0 to $6 \mathrm{~h}$, followed by a drop in the expression level at $24 \mathrm{~h}$. The most abundant group was the B73 Cluster 2 , with 628 genes showing a negative slope from 0 to $6 \mathrm{~h}$, and then returned to the basal levels at the $24 \mathrm{~h}$ time point. B73 Cluster 3 had 191 genes whose expression showed a positive slope from 0 to $24 \mathrm{~h}$. In Mo17, 958 transcripts were clustered into two groups (Figure 4A, Table S4). Cluster 1 contained 617 genes whose expression showed a positive slope from 0 to $6 \mathrm{~h}$, followed by a drop in the expression level at $24 \mathrm{~h}$, and Cluster 2 contained 341 genes whose expression showed an initial drop from 0 to $6 \mathrm{~h}$, followed by reversion to the basal levels at $24 \mathrm{~h}$ (Figure 4A, Table S4). To reveal the different biological processes in each gene expression cluster, pathway enrichment analysis was performed using MetGenMAP (Joung et al., 2009). Pathways that were enriched among the transcripts in $R$. padi-treated B73 included (1) many plant defense- and stress response-related genes (Figure 4B, Table S4), such as benzoate degradation II in Cluster 1; (2) proline degradation, tryptophan biosynthesis, and chlorogenic acid biosynthesis I in Cluster 2; (3) photorespiration, Rubisco shunt, glycine biosynthesis, phospholipid desaturation, and glycolipid desaturation, which were mostly related to primary metabolism in Cluster 3 . Notably, defense-related salicylate biosynthesis and chlorogenic acid biosynthesis could be found in Cluster 1, and DIMBOAglucoside degradation was in Cluster 2 (Figure 4B, Table S4).

In order to gain further insight into the regulatory mechanism underlying the differences between the responses of B73 and Mo17 to $R$. padi on the gene expression level, differentially regulated TFs were identified. It was found that $R$. padi herbivory specifically regulated 35 and 14 TFs in B73 and Mo17, respectively, and 9 TFs were regulated in common (Figure 5A, Table S5). We found that the 44 TFs distributed in seven families (MYB, WRKY, b HLH, NAC, GRAS, HSF, and Dof) in B73, most of which were down-regulated (Figure 5B, Table S5). In contrast, the 23 TFs in Mo17 distributed in five families (MYB, WRKY, b HLH, NAC, and GRAS), and most of them were up-regulated (Figure 5C, Table S5). However, 7 out of the 9 




FIGURE 1 | The resistance levels of different maize lines to $R$. padi. (A) The survival rates of $R$. padi on B73, Zong3, Chang7-2, A188, and Mo17. (B) The life span and the time needed from birth to first reproduction. (C) Fecundity of $R$. padi on B73 and Mo17. Values $=$ means \pm SE; $n=20$; one-way ANOVA was used in (A), t-test was applied in (B,C); $\left.{ }^{\star} P<0.05 ;{ }^{\star \star} P<0.01\right)$.

commonly regulated TFs in B73 and Mo17 were down-regulated in B73 but were up-regulated in Mo17 (Figure 5D, Table S5).

\section{Clustering of Expression Patterns in B73 and Mo17}

Given the large gene expression differences between B73 and Mo17 in response to $R$. padi feeding, we expected that these two lines may also show a distinct transcript pattern at individual time points.

We compared the DEGs between B73 and Mo17 under the control condition and after 6 and $24 \mathrm{~h}$ of $R$. padi feeding, and it was found that 2,174 genes were always differentially expressed under all these conditions, but specifically, 1,437, 496, and 748 genes exhibited different expression levels only under control conditions and after 6 and $24 \mathrm{~h}$ of $R$. padi feeding, respectively (Figure 6A, Table S6).

Specifically, (1) the DEGs between B73 0 and Mo17 $0 \mathrm{~h}$ were clustered to 11 pathways, most of which were associated with primary metabolism, except salicylate biosynthesis and chlorogenic acid biosynthesis I (Figure 6B, Table S6); (2) DEGs between B73 and Mo17 at $6 \mathrm{~h}$ were linked to four defenserelated pathways, which were phenylpropanoid biosynthesis, chlorogenic acid biosynthesis I, tyrosine biosynthesis, and phenylalanine biosynthesis; (3) DEGs between B73 and Mo17 at $24 \mathrm{~h}$ were enriched on five pathways: Proline biosynthesis I, Abscisic acid biosynthesis, Proline biosynthesis III, Arginine biosynthesis IV, and Citrulline biosynthesis (Figure 6B, Table S6). Thus, B73 and Mo17 showed different response to R. padi feeding in gene expression pattern. More detailed transcriptomic comparisons between B73 and Mo17 over time are shown in Table S6.

We next determined the transcript levels of TFs in B73 and Mo17 under control condition, 6 and $24 \mathrm{~h}$ after $R$. padi treatment (Figure 7, Table S7). Despite the large differences between the transcriptomes of B73 and Mo17, only 96 TFs were found to have different expression levels between B73 and Mo17 under the control condition, while 62 and 57 TFs were differentially expressed between these two lines after being treated with aphid feeding for 6 and $24 \mathrm{~h}$, respectively (Figure 7A, Table S7); and among these, 38,12 , and $11 \mathrm{TF}$ genes were specifically different only in the control samples and those induced by 6 and $24 \mathrm{~h}$ of $R$. padi herbivory. Only 28 TFs were differentially expressed between B73 and Mo17 at all time points, and these TFs showed the same patterns of regulation (Figure 7B, Table S7). The entirety of TFs that were differentially expressed between B73 and Mo17 were distributed in WRKY, MYB, NAC, b HLH, C2H2, GRAS, HSF, Dof, and b ZIP (Figure 7C, Table S7). Under all conditions, most TFs of the WRKY, MYB, NAC, b HLH, and $\mathrm{C} 2 \mathrm{H} 2$ group had greater expression levels in B73 than in Mo17. 


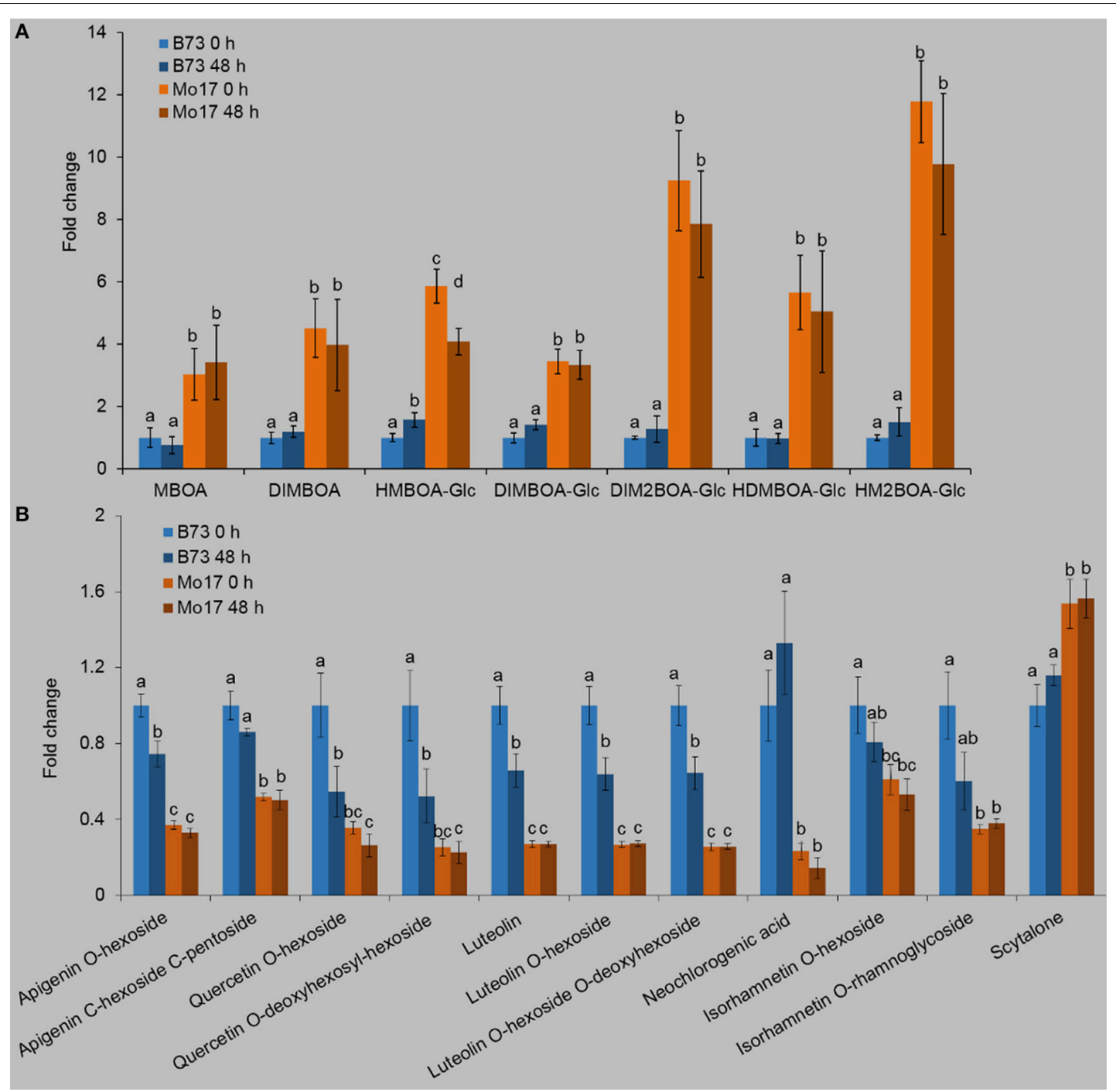

FIGURE 2 | Profiles of Bxs and phenolics in B73 and Mo17. Maize leaves were treated with $R$. padi herbivory, and samples were collected at $48 \mathrm{~h}$; non-treated leaves severed as controls $(\mathrm{O} h)$. Relative contents of Bxs (A) and phenolics (B) in B73 and Mo17. Values = means $\pm \mathrm{SE} ; n=5$; different letters above bars indicate significant differences (one-way ANOVA and Duncan's multiple range test; $P<0.05$ ); all values of the B73 group are normalized to 1 . Complete data can be found in Table $S 1$.

Notably, the number of WRKY, MYB, and NAC TFs, whose transcript levels were higher in B73 than in Mo17, decreased after R. padi feeding; for example, in B73 $0 \mathrm{~h}$ samples, 19 WRKYs had higher expressions than in Mo17, but in B73 6 and B73 $24 \mathrm{~h}$ the number of TFs that had higher expression in B73 decreased to 9 and 12, respectively (Figure 7C). It is likely that TFs may have played an important role in shaping the differences of B73 and Mo17 transcriptomes under control and R. padi-induced conditions.

\section{Correlation between Transcription Factors and Bx Biosynthesis Genes}

In order to understand the possible genetic basis for the different levels of Bxs in B73 and Mo17, the transcriptome data were screened for Bx biosynthesis-related genes that had been annotated (http://www.maizegdb.org/), and the newly reported
BX13 and BX14 (Handrick et al. (2016). Most of the Bx biosynthetic genes had different expression levels between B73 and Mo17 (Table S7). Importantly, BX1 and BX13 levels were much higher in Mo17 than in B73 (at least 7.8 and 33 times greater in Mo17 than in B73) in all samples (Figures 8A,B, Table S7). BX1 is very important for Bx biosynthesis (Butron et al., 2010); without the expression of BX13, DIM 2 BOA-Glc cannot be detected, suggesting $B X 13$ plays a significant role in Bx biosynthesis (Handrick et al., 2016). As Bx levels, such as $\mathrm{DIM}_{2} \mathrm{BOA}-\mathrm{Glc}$ and HDMBOA-Glc, were much higher in Mo17 than in B73 (Figure 2A), BX1 and BX13 likely accounted for the high Bx contents in Mo17.

Only four TFs, a MYB (GRMZM2G108959), a GRAS (GRMZM2G015080), a NAC (GRMZM2G179049), and a WRKY (GRMZM2G425430), showed greater expression levels in Mo17 than in B73 under all conditions (Figure 8C, Table S7), 


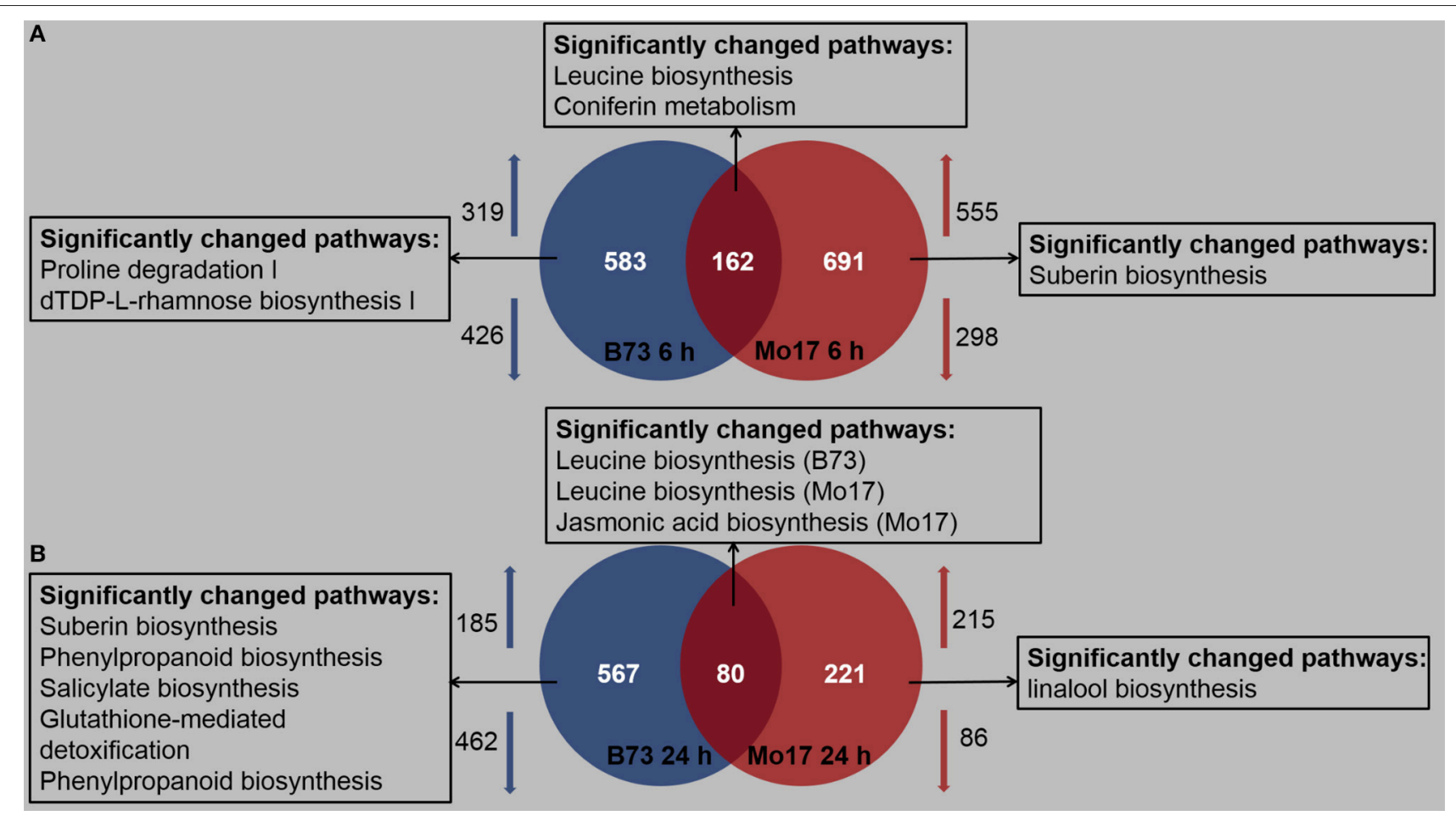

FIGURE 3 | Profiles of regulated maize transcripts after 6 and $24 \mathrm{~h}$ of $R$. padi infestation on B73 and Mo17. The fourth maize leaves were each treated with 50 aphids, and samples were collected at 6 and $24 \mathrm{~h}$; untreated leaves served as controls. Overall transcriptionally regulated genes and functional distribution of differentially expressed unique genes (fold change $>2, P<0.05$ ) after $6 \mathrm{~h}(\mathbf{A})$ and $24 \mathrm{~h}(\mathbf{B})$ of $R$. padi herbivory in B73 and Mo17. Arrows indicate up- and down-regulated genes, respectively. Complete data can be found in Table S3.

suggesting that these four TFs may play a role in regulating the expression of $B X 1$ and $B X 13$, resulting in the high $\mathrm{Bx}$ levels in Mo17.

\section{Differentially Expressed Volatile Terpene Biosynthesis Genes}

Terpene synthases (TPSs) catalyze the formation of diverse volatile terpenes (Bohlmann et al., 1998; Cheng et al., 2007; Dudareva et al., 2013). To investigate whether the volatile terpene biosynthesis pathways respond to $R$. padi feeding, we screened for the expression levels of 11 TPS genes in B73 and Mo17 that had been annotated by the Plant MetGenMAP to be 10 sesquiterpene synthases (TPS1 to 10) and one monoterpene synthase (GRMZM2G030583) (Joung et al., 2009). Among these 11 TPS genes inspected, TPS8 (GRMZM2G038153) was induced (1.93- and 1.11-fold increased at 6 and $24 \mathrm{~h}$, respectively) by $R$. padi infestation while TPS10 was repressed (84 and $43 \%$ decreased after 6 and $24 \mathrm{~h} R$. padi feeding, respectively) in B73, but not in Mo17 (Figure S2A, Table S8). TPS2 (GRMZM2G046615) and TPS3 (GRMZM2G064406) were 4.98and 3.91-fold up-regulated by R. padi herbivory after $6 \mathrm{~h} R$. padi feeding in Mo17, but not in B73 (Figure S2B, Table S8). We further compared the expression level of TPS genes between B73 and Mo17. Without $R$. padi feeding, there were five genes (TPS2, TPS3, TPS7, TPS8, and TPS10) differentially expressed between these two lines: TPS2, TPS3, TPS8, and TPS10 showed higher levels in B73 compared to those in Mo17, while TPS7 showed the opposite pattern (Figure S3A, Table S8). After 6 h of R. padi feeding, only TPS7 and TPS 8 showed different expression levels between B73 and Mo17 (Figure S3B, Table S8), and after $24 \mathrm{~h}$ of $R$. padi feeding TPS7 still expressed higher in B73 while the expression level of TPS10 was lower in B73 than in Mo17 (Figure S3C, Table S8).

\section{Differentially Spliced Genes in B73 and Mo17}

The differentially spliced genes (DSGs) were investigated to determine whether AS might be involved in the response of B73 and Mo17 to $R$. padi feeding. $6 \mathrm{~h}$ after $R$. padi feeding on B73, 36 DSGs were identified, and there was no overlap between DEGs and DSGs at this time point (Figure 9A, Tables S3, S9); only one gene (among 59 DSGs) was both a DEG and a DSG after $24 \mathrm{~h}$ of $R$. padi feeding on B73 (Figure 9B, Tables S3, S9); among these 36 and 59 DSGs, only two genes showed changes in AS in B73 at both 6 and $24 \mathrm{~h}$ after $R$. padi feeding (Figure 9C, Table S9). Similarly, 59 and 39 DSGs were found in Mo17 after 6 and $24 \mathrm{~h}$ of $R$. padi feeding, respectively (Figure 9F, Table S9), and only a few genes were both regulated on the expression and AS level (Figures 9D,E, Tables S3, S9). Furthermore, six genes were found to be common between the DSGs identified in the samples of Mo17 6 and Mo17 24 h (Figure 9F, Table S9). To gain further insight into $R$. padi-induced dynamic AS responses in 

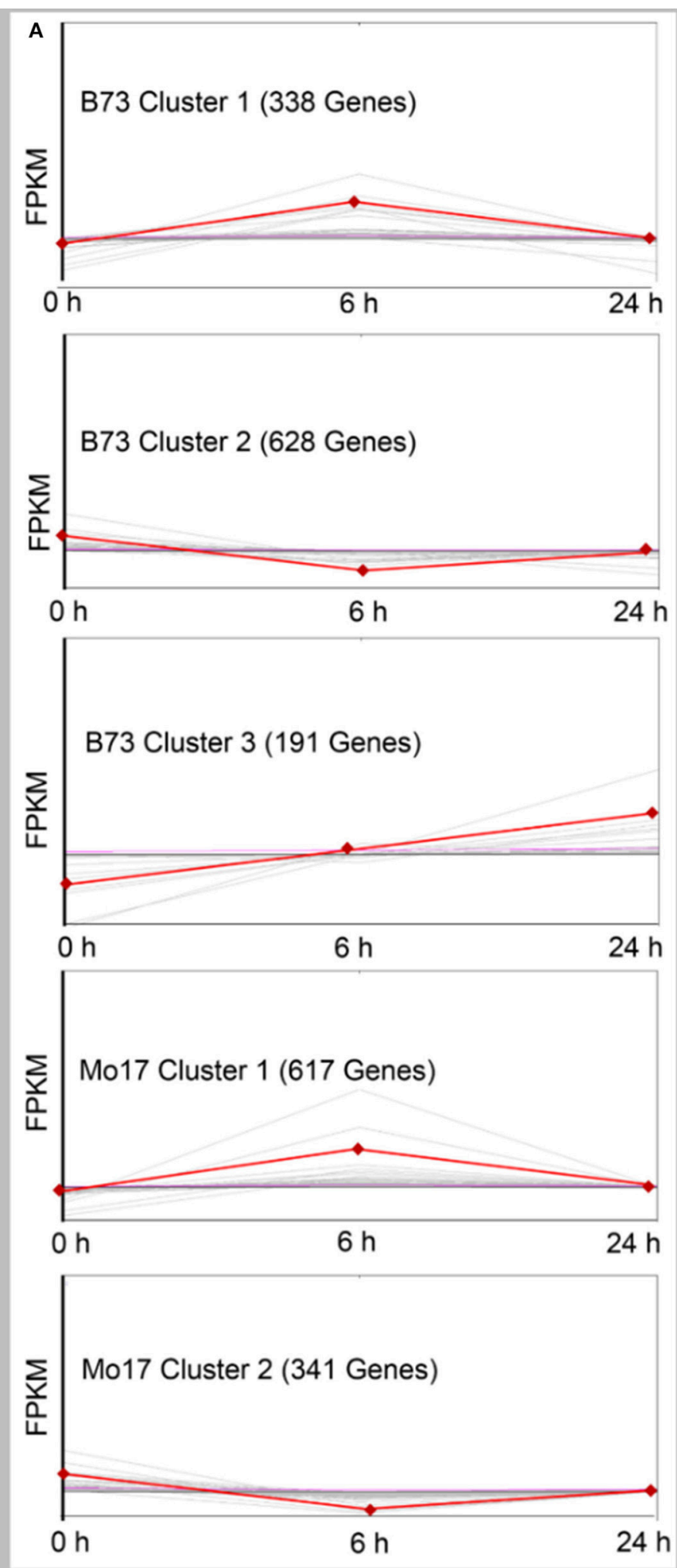

\begin{tabular}{|c|c|c|}
\hline B & B73 Cluster 1 (338 Genes) & $P$-value \\
\hline & Leucine biosynthesis & $1.65 \mathrm{E}-04$ \\
\hline & Benzoate degradation II (aerobic and anaerobic) & 8.89E-03 \\
\hline & $\mathrm{Fe}$ (III)-reduction and $\mathrm{Fe}$ (II) transport & 8.89E-03 \\
\hline & CDP-diacylglycerol biosynthesis I & $3.00 \mathrm{E}-02$ \\
\hline & CDP-diacylglycerol biosynthesis II & $3.00 \mathrm{E}-02$ \\
\hline & Phosphatidylglycerol biosynthesis I (plastid) & $3.96 \mathrm{E}-02$ \\
\hline & Triacylglycerol biosynthesis & $3.96 \mathrm{E}-02$ \\
\hline & Phosphatidylglycerol biosynthesis II & $3.96 \mathrm{E}-02$ \\
\hline & B73 Cluster 2 (628 Genes) & $P$-value \\
\hline & Proline degradation I & 8.34E-04 \\
\hline & Dtdp-L-rhamnose biosynthesis I & 1.65E-03 \\
\hline & $\begin{array}{l}\text { Superpathway of phenylalanine, tyrosine, and } \\
\text { tryptophan biosynthesis }\end{array}$ & $3.49 \mathrm{E}-03$ \\
\hline & Tryptophan biosynthesis & $3.64 \mathrm{E}-03$ \\
\hline & Chlorogenic acid biosynthesis II & 7.17E-03 \\
\hline & Sucrose degradation I & $9.00 \mathrm{E}-03$ \\
\hline & O-antigen biosynthesis ( $E$. Coli $)$ & 9.38E-03 \\
\hline & Chlorogenic acid biosynthesis I & $1.22 \mathrm{E}-02$ \\
\hline & UDP-D-xylose biosynthesis & 1.67E-02 \\
\hline & Proline degradation II & $1.70 \mathrm{E}-02$ \\
\hline & Sucrose degradation III & $2.20 \mathrm{E}-02$ \\
\hline & Xylan biosynthesis & $2.57 \mathrm{E}-02$ \\
\hline & Divinyl ether biosynthesis II (13-LOX) & $2.90 \mathrm{E}-02$ \\
\hline & 13-LOX and 13-HPL pathway & $2.90 \mathrm{E}-02$ \\
\hline & Coniferin metabolism & $3.36 \mathrm{E}-02$ \\
\hline & Methionine degradation II & $3.36 \mathrm{E}-02$ \\
\hline & Phenylpropanoid biosynthesis, initial reactions & $3.62 \mathrm{E}-02$ \\
\hline & Phenylpropanoid biosynthesis & $3.80 \mathrm{E}-02$ \\
\hline & Simple coumarins biosynthesis & 3.93E-02 \\
\hline & B73 Cluster 3 (191 Genes) & $P$-value \\
\hline & Photorespiration & $3.65 \mathrm{E}-03$ \\
\hline & Rubisco shunt & $1.03 \mathrm{E}-02$ \\
\hline & Glycine biosynthesis IV & 2.16E-02 \\
\hline & Glycolipid desaturation & 4.29E-02 \\
\hline & Phospholipid desaturation & 4.29E-02 \\
\hline & Glycine biosynthesis I & $4.98 \mathrm{E}-02$ \\
\hline & Mo17 Cluster 1 (617 Genes) & $P$-value \\
\hline & Suberin biosynthesis & 1.35E-05 \\
\hline & Wax esters biosynthesis I & 4.26E-04 \\
\hline & Leucine biosynthesis & $2.15 \mathrm{E}-03$ \\
\hline & Salicylate biosynthesis & 3.33E-03 \\
\hline & Asparagine degradation I & 4.16E-03 \\
\hline & Phenylpropanoid biosynthesis, initial reactions & $5.71 \mathrm{E}-03$ \\
\hline & Triacylglycerol biosynthesis & $6.20 \mathrm{E}-03$ \\
\hline & Phenylpropanoid biosynthesis & $2.01 \mathrm{E}-02$ \\
\hline & $\mathrm{Fe}$ (III)-reduction and $\mathrm{Fe}$ (II) transport & $2.10 \mathrm{E}-02$ \\
\hline & Ethylene biosynthesis from methionine & 2.11E-02 \\
\hline & Chlorogenic acid biosynthesis I & 2.17E-02 \\
\hline & Very long chain fatty acid biosynthesis & $2.38 \mathrm{E}-02$ \\
\hline & Mo17 Cluster 2 (341 Genes) & $P$-value \\
\hline & Coniferin metabolism & $1.61 \mathrm{E}-02$ \\
\hline & Sucrose degradation I & $1.79 \mathrm{E}-02$ \\
\hline & Free phenylpropanoid acid biosynthesis & $3.19 \mathrm{E}-02$ \\
\hline & Methylquercetin biosynthesis & 3.19E-02 \\
\hline & Sucrose degradation III & 3.31E-02 \\
\hline & DIMBOA-glucoside degradation & $3.49 \mathrm{E}-02$ \\
\hline & UDP-N-acetyl-D-glucosamine biosynthesis I & $3.98 \mathrm{E}-02$ \\
\hline & Simple coumarins biosynthesis & 4.99E-02 \\
\hline
\end{tabular}

FIGURE 4 | Overview of gene expression trends and clusters in R. padi-treated B73 and Mo17. (A) A total of 1157 transcripts in B73 (in three clusters) and 958 transcripts in Mo17 (in two clusters) with significant expression profile changes for at least one time-point after the $R$. padi feeding. The total number of transcripts in each cluster is indicated in the brackets, and the data for individual genes are shown in light gray. Average expression values for each cluster are shown in red. All genes selected for this analysis have significant differences of 1 -fold (up- or down-regulated), $P<0.05$. (B) Pathway enrichment analysis of each cluster using MetGenMAP to identify metabolic functions that were regulated. Full descriptions can be found in Table S4. 




FIGURE 5 | Differentially regulated transcription factors in B73 and Mo17 after $R$. padi herbivory. The fourth maize leaves were each treated with 50 aphids, and samples were collected at 6 and $24 \mathrm{~h}$ after $R$. padi treatment; untreated leaves served as controls. (A) Venn diagram depicting the number of transcription factors (TFs) significantly changed in B73 and Mo17 after 6 and $24 \mathrm{~h}$ of $R$. padi treatment. Heat maps indicate relative expression levels (fold change after log 2 transformation) of TFs regulated by R. padi feeding in B73 (B) and Mo17 (C). (D) Relative expression levels (fold change after log 2 transformation) of the 9 common regulated TFs in B73 and Mo17. Detailed data can be found in Table S5.

B73 and Mo17, pathway enrichment analysis was performed on the DSGs using MetGenMAP (Joung et al., 2009). No grouping of the DSGs in B73 6 and B73 $24 \mathrm{~h}$ (Figure S4, Table S9) into enriched pathways showed no overlapping function. Notably, three pathways were in common among the enriched pathways in the DSGs of Mo17 6 and Mo17 24 h: synthesis of suberin, 


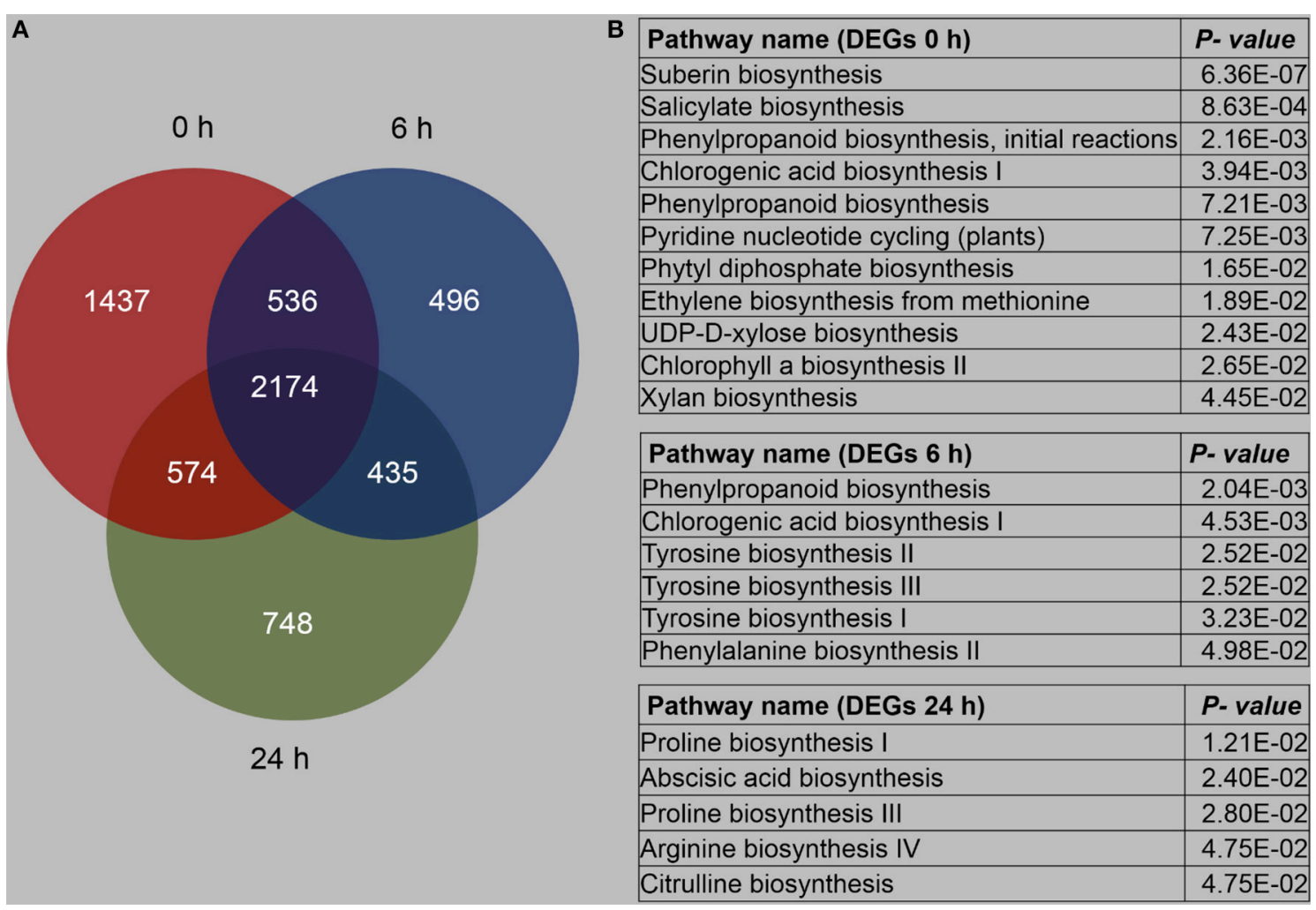

FIGURE 6 | Overview of differentially expressed genes between B73 and Mo17. The fourth maize leaves were each treated with 50 aphids, and samples were collected at 6 and 24 h; untreated leaves served as controls (0 h). (A) Number of genes with differentially expressed levels between B73 and Mo17. (B) Pathway enrichment analysis using MetGenMAP to identify metabolic functions that were differentially expressed between B73 and Mo17. Full descriptions can be found in Table S6.

salicylate biosynthesis, and phenylpropanoid biosynthesis, and these are all defense-related (Figure S4, Table S9). These results indicate that B73 and Mo17 respond specifically to $R$. padi feeding on the AS level.

In order to gain further insight into the differences between these two maize lines, the DEGs and DSGs between B73 and Mo17 under control condition and at 6 and $24 \mathrm{~h}$ after $R$. padi treatment were determined. Consistent with the large differences in gene expression levels between these lines, 688 DSGs were identified between B73 0 and Mo17 $0 \mathrm{~h}$, and 159 genes were regulated both on expression and AS level (Figure 10A, Tables S6, S10). Similarly, after 6 and $24 \mathrm{~h}$ of R. padi infestation, B73 and Mo17 had 595 and 658 DSGs, respectively, and 99 and 122 genes were both DEGs and DSGs (Figures 10B,C, Tables S6, S10). The enriched pathways in the DSGs between B73 and Mo17 under control and after $6 \mathrm{~h}$ of $R$. padi feeding included flavin, spermine, and spermidine biosynthesis; terpenoid biosynthesis is the most enriched pathway in the DSGs identified between B73 and Mo17 after 24 h of $R$. padi herbivory (Figure S5, Table S10). Comparison of all these three groups of DSGs indicated that there were 181, 126, and 166 condition-specific DSGs between B73 and Mo17, while 340 genes were found consistently differentially spliced under all conditions (Figure 10D, Table S10). Furthermore, 10 TFs were found among these 340 DSGs, which belonged to the families of WRKY, bZIP, b HLH, GRAS, MYB, C2H2, MYB, and GATA (Table S10), and notably, none of these TFs showed differences in their expression levels between these two maize lines.

These results suggest that $R$. padi feeding induces specific AS patterns in different maize lines. Furthermore, AS difference also existed between B73 and Mo17 and is largely independent of the regulation of gene expression levels.

\section{DISCUSSION}

Within a species, phenotypic variations caused by heritable genetic diversity are the driving force in adaptation to the ever-changing environment. Like many other traits, phenotypic variations of resistance to insects has been demonstrated to vary among populations of wild plants (Wu et al., 2008) and varieties of crops (Meihls et al., 2013; Wang et al., 2014; Tzin et al., 2015b). Crop plants, such as maize, have rich resources of genetic diversity due to thousands of years of artificial and natural selection. These diverse genetic crop resources are not only important for breeding new varieties with improved agronomic and resistance traits, but are also the material for scientists studying the mechanisms underlying phenotypic variations. 


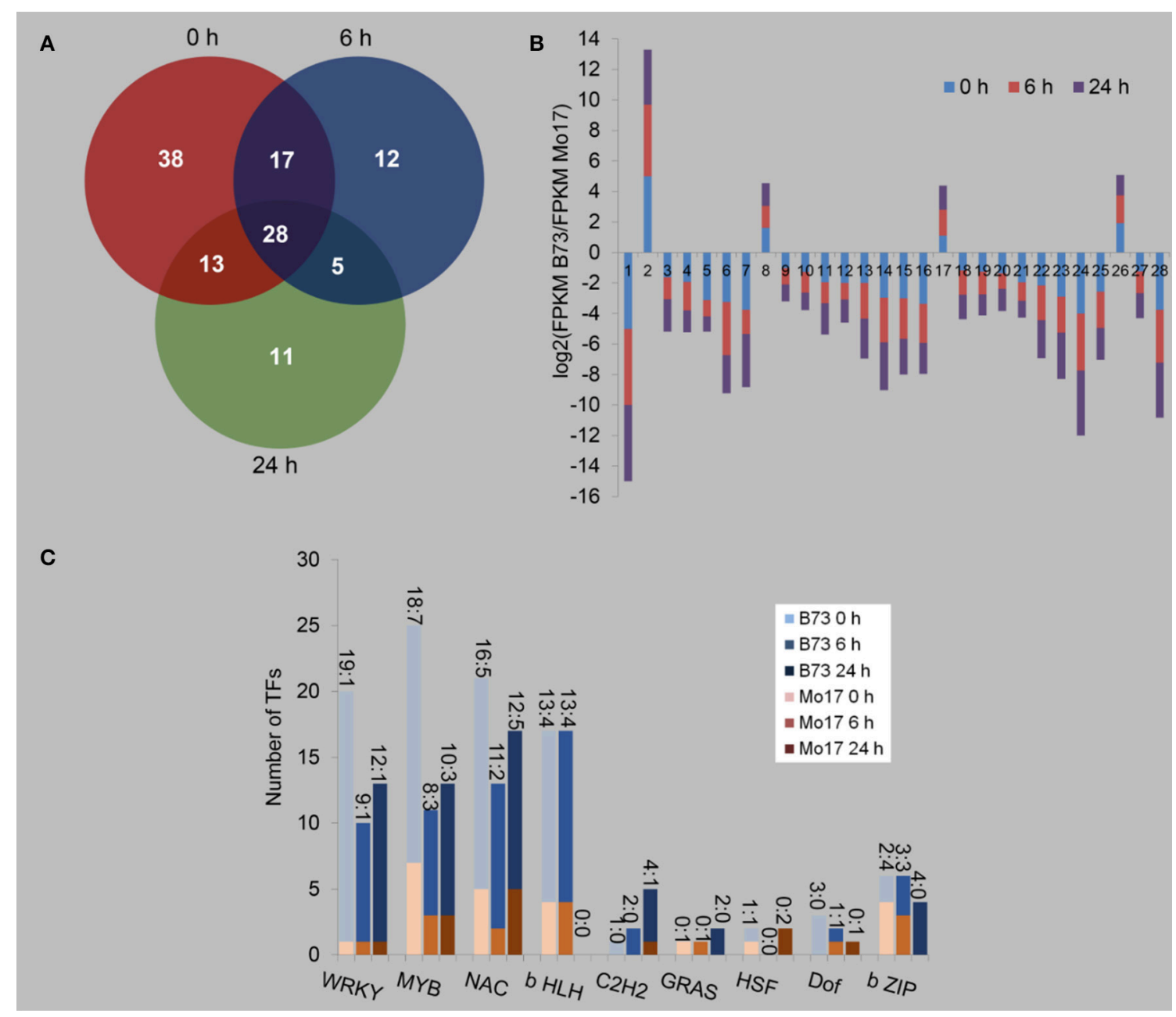

FIGURE 7 | Differentially regulated transcription factors between B73 and Mo17 with and without $R$. padi treatment. The fourth maize leaves were each treated with 50 aphids, and samples were collected at 6 and $24 \mathrm{~h}$ (untreated leaves served as controls, depicted as $0 \mathrm{~h}$ ). (A) Venn diagram indicating the number of differently expressed transcription factors (TFs) between B73 and Mo17 at each time point. (B) Expression profiles of the 28 common regulated TFs (X-axis represent randomly assigned gene numbers, and their gene IDs are in Table S7). (C) Distribution of the families of TF differentially regulated between B73 and Mo17, in control, at 6 and $24 \mathrm{~h}$ of $R$. padi herbivory-treated samples. On top of each bar, the number before the colon indicates the number of TFs that had greater expression in B73 than in Mo17, and the number after the colon indicates the number of TFs whose expression were lower in B73 than in Mo17. Full descriptions can be found in Table S7.

Many lines of evidence have indicated the importance of Bxs defense against insects. In maize, Bx levels are negatively correlated with the growth of the grain aphid Sitobion avenae and $R$. padi (Thackray et al., 1990), supplementation of Bxs to artificial diet increased the mortality of the greenbug Schizaphis graminum (Argandona et al., 1983), and Bxs are also important for the defense against the European Corn Borer Ostrinia nubilalis (Niemeyer, 2009). Our detailed analyses in control and R. padi-induced B73 and Mo17 indicate that Bx biosynthesisrelated genes and $\mathrm{Bx}$ accumulation are not regulated by $R$. padi feeding. This is consistent with the finding that $R$. maidis herbivory on B73 did not change the concentrations of HMBOAGlc, DIM ${ }_{2}$ BOA-Glc, and HDMBOA-Glc (Meihls et al., 2013), although $B X 1, B X 2, B X 6$, and $B X 7$ showed transient increases (Tzin et al., 2015a). We found that the transcript levels of $B X 1$ and $B X 13$ are substantially greater in Mo17 than in B73. BX1 is the initial enzyme in the Bx biosynthesis pathway, and genetic analysis suggested that the high expression level of the $B X 1$ in Mo17 very likely accounts for its abundant Bx content (Zheng et al., 2015). Recently, Handrick et al. (2016) identified BX13 as a 2-oxoglutarate-dependent dioxygenase, encoding an enzyme that converts DIMBOA-Glc to TRIMBOA-Glc, and maize lines having a mutation in BX13 displayed compromised resistance to the leaf aphid $R$. maidis. Therefore, the high expression levels of $B X 1$ and $B X 13$ in Mo17 are very likely the reason for its high contents of Bxs and thus high resistance to R. padi (Figure 11). The cis- and trans-elements of $B X 1$ and $B X 13$, such as TFs and TF-binding sites in the promoters, need to be studied to reveal the underlying mechanism by which these plants maintain their high expression of these two BX-biosynthetic genes (Figure 11).

In contrast to Bxs, most detected phenolic and flavonoid metabolites showed higher contents in B73 than in Mo17. Wheat 


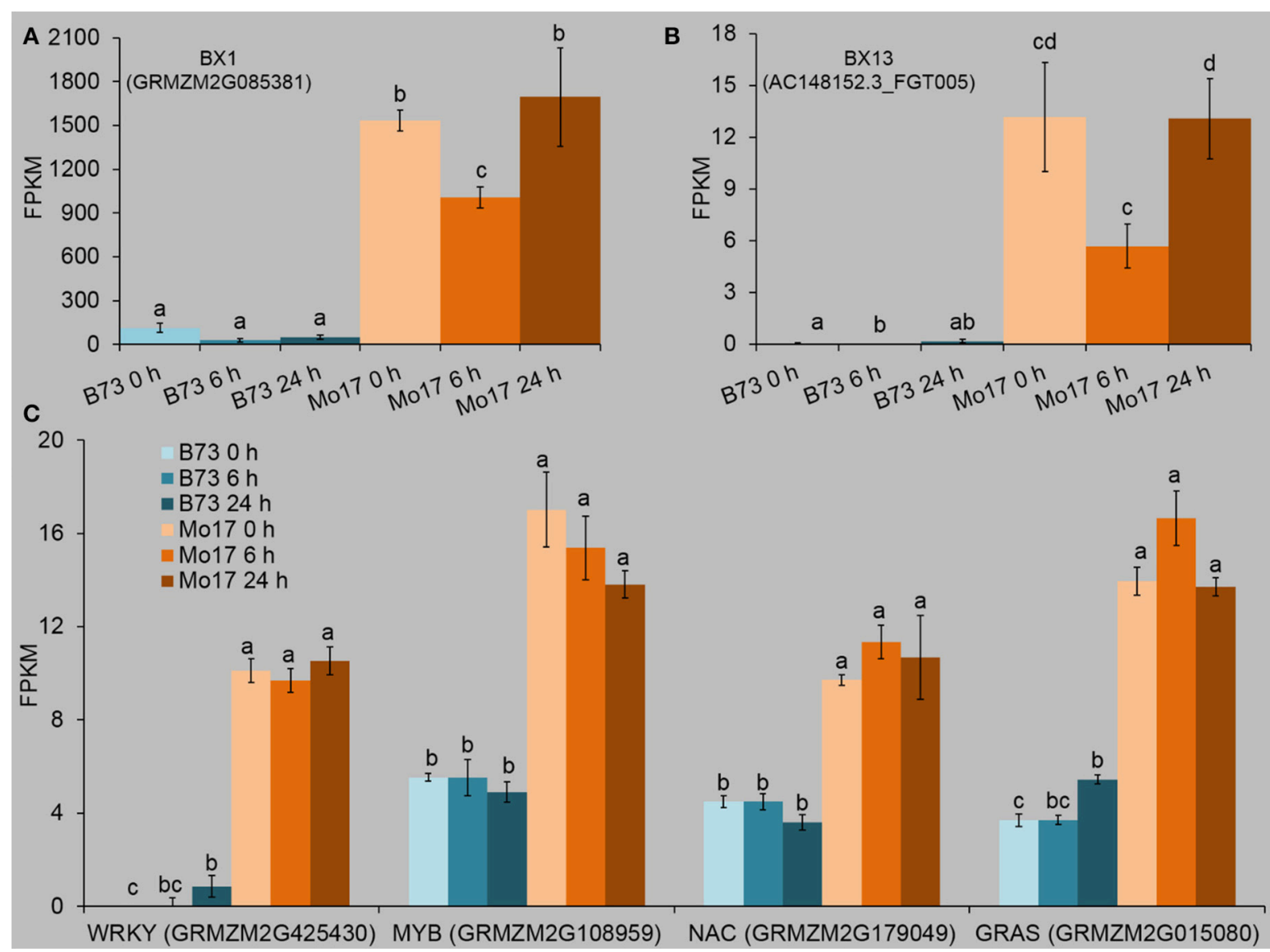

FIGURE 8 | Expression profiles of Bx biosynthetic genes and transcription factors whose expression patterns were correlated with that of BX1. The fourth maize leaves were each treated with 50 aphids per plant, and samples were collected at 6 and $24 \mathrm{~h}$ after $R$. padi treatment (untreated leaves served as controls, depicted as 0 h). Relative changes of the transcript levels of $B \times 1(\mathbf{A})$ and $B \times 13$ (B). Data were extracted from the FPKM values of the target genes in the RNA-seq datasets. (C) Transcription factors (TFs) which showed similar expression patterns to that of $B X 1$. Values $=$ means \pm SE; $n=3$; different letters above bars indicate significant differences (one-way ANOVA Duncan's multiple range test; $P<0.05$ ). Full descriptions can be found in Table S7.

cultivars with high concentrations of soluble and cell wall-bound phenolics were much less attractive to $R$. padi than the cultivars with low phenolic concentrations (Leszczynski et al., 1985), and another study showed that phenolics in triticale hybrids were negatively associated with the attractiveness to $R$. padi (Wójcicka, 2010). Notably, R. padi aphid feeding led to decrease of phenolics in B73, but had no effect on most phenolics in Mo17. More studies are needed to clarify whether phenolics are involved in the defense against $R$. padi in maize.

Tzin et al. (2015a) found that on the maize tps $2 / 3:: D s$ mutant, $R$. maidis reproduction was decreased by $\sim 73 \%$ compared to the reproduction of aphids on the wild-type W22, suggesting that the terpene products of TPS 2 and TPS 3 may promote the growth of aphids as feeding stimulants (Tzin et al., 2015a). We found that TPS2 and TPS3 showed higher expression levels in B73 than in Mo17 under normal condition (Figure S3A, Table S8). Thus, it is possible that B73 contains greater levels of TPS2/3-produced feeding stimulants (terpenes) than does Mo17, which partly accounts for the susceptibility of B73 to aphids. TPS10 catalyzes the formation of $(E)-\beta$ farnesene, $(E)$ - $\alpha$-bergamotene, and sesquiterpene hydrocarbons, and overexpression of TPS10 protect plants by attracting the parasitoid (C. marginiventris) of lepidopteran insects (Schnee et al., 2006). Even though TPS10 expression was suppressed by $R$. padi feeding in B73 (not in Mo17), B73 always exhibited greater levels of TPS10 than did Mo17 (Figure S3, Table S8), suggesting that B73 may have better indirect defenses than does Mo17. Whether the terpene products of TPS10 may also act as $R$. padi feeding stimulants remains unknown. Moreover, TPS7, which participates in sesquiterpene biosynthesis, consistently showed higher levels in Mo17 than in B73 (Figure S3, Table S8), and this may also partly contribute to the different resistance to $R$. padi in these two maize lines. Further analysis of the contents of terpenes in different maize lines and the functions of individual terpenes in attracting the aphid predators (such as parasitoids) or stimulating/deterring aphid feeding are needed for further understanding the correlation between the 


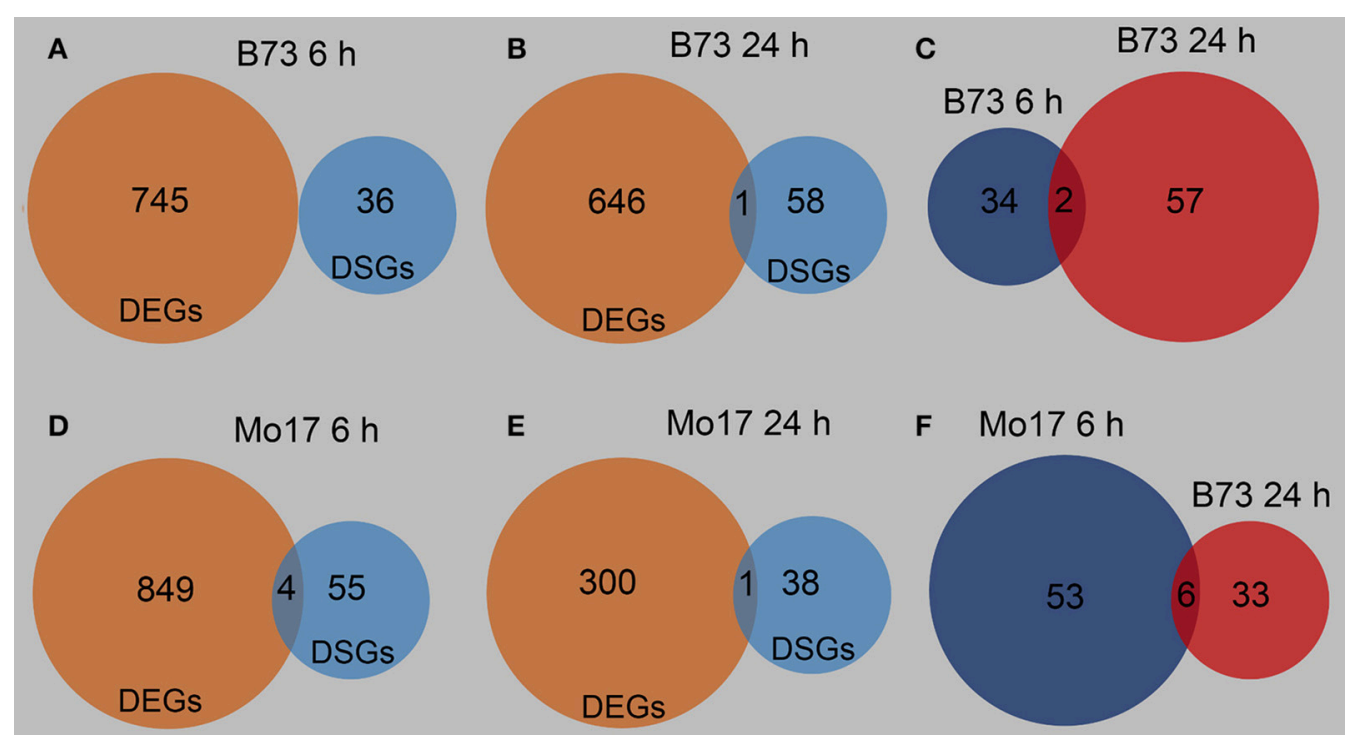

FIGURE 9 | Overview of the differentially spliced genes in B73 and Mo17. The fourth maize leaves were each treated with 50 aphids per plant, and samples were collected at 6 and $24 \mathrm{~h}$ after $R$. padi treatment; untreated leaves served as controls. Venn diagrams indicating differentially expressed genes (DEGs) and differentially spliced genes (DSGs) at $6 \mathrm{~h}$ (A) and $24 \mathrm{~h}$ (B) of $R$. padi feeding in B73. Venn diagram showing specific and overlapping DSGs in B73 at 6 and $24 \mathrm{~h}$ of $R$. padi feeding (C). Venn diagrams indicating DEGs and DSGs at $6 \mathrm{~h}$ (D) and $24 \mathrm{~h}$ (E) of $R$. padi feeding on Mo17. Venn diagram showing overlapping DSGs in Mo17 at 6 and $24 \mathrm{~h}$ of R. padi feeding (F). Full descriptions can be found in Tables S3, S9.

differentially expressed TPSs and cultivaral differences of aphid resistance.

A recent transcriptomic study on $R$. maidis-induced $\mathrm{B} 73$ allows to compare the responses of $\mathrm{B} 73$ to $R$. maidis (Tzin et al., 2015a) and R. padi (this study). Most of the significantly changed transcripts after $R$. maidis feeding were up-regulated (Tzin et al., 2015a), whereas $R$. padi herbivory transcriptionally suppressed rather than induced genes. More than 1,600 transcripts, whose expression profile changed at least at one time-point after $R$. maidis feeding clustered into six groups; the biological processes included the shikimate pathway, biosynthesis of aromatic amino acids, salicylic acid, jasmonic acid, auxin, and ethylene biosynthesis from Met. More than 1,100 genes induced by $R$. padi feeding were clustered to three groups, whose biological processes included chlorogenic acid biosynthesis I and chlorogenic acid biosynthesis II. In parallel to the transcriptomic profiles, $R$. maidis feeding up-regulated most of the metabolites in B73, but our data showed that most detected metabolites in R. padi-infested B73 did not change significantly (Figure 2, Table S1). Thus, similar to Miscanthus sinensis, which was found to be resistant to $R$. padi but susceptible to $R$. maidis (Huggett et al., 1999), B73 responded distinctive to $R$. maidis and $R$. padi, and this was likely because of the different aphid species used, but could also be related to different treatments ( 10 adult $R$. maidis and 50 adult $R$. padi) or plant growth stages (2- and 3-week-old seedlings, respectively).

B73 and Mo17 responded to $R$. padi feeding with high specificity on the transcriptomic level; For instance, most of the TFs in B73 were down-regulated by $R$. padi herbivory, while most of those in Mo17 were up-regulated (Figure 11). Importantly, these large transcriptomic differences were independent of $R$. padi feeding. In line with this, we found more differentially expressed TF between B73 and Mo17 under any condition than there were differentially expressed TF induced by $R$. padi herbivory in these lines. All these data are consistent with the large genetic differences between maize inbreed lines (Springer et al., 2009; Jiao et al., 2012). Although thousands of genes were differentially expressed between B73 and Mo17, only a small number (at most 96 in the control samples) were TFs. It is likely that at least some of these TFs, if not all, accounted for the transcriptomic differences. Using genetic mapping and allelespecific transcript measurements, Zheng et al. (2015) found that both cis-element(s) and trans-acting factors affect BX1 transcript levels in Mo17. Our analysis indicated that only four differentially expressed TFs, a WRKY, a MYB, a NAC, and a GRAS gene, showed a good correlation with the $B X 1$ expression patterns in B73 and Mo17 (Figure 8, Table S7). Moreover, emerging evidence has pointed to the role of AS in regulating TF activity (Seo et al., 2013). In addition to these four TFs that have constantly different expression levels between B73 and Mo17, 10 other TFs were found to be differentially spliced between B73 and Mo17 under all conditions (Table S10). It is possible that, among these 14 differentially expressed or spliced TFs, some influence the expression of $B X 1$ and further affect the content of Bxs. Functional studies focusing on these TFs are needed to elucidate whether they play a role in regulating $\mathrm{Bx}$ biosynthesis.

Alternative splicing (AS) is highly prevalent in plants, and is known to rapidly change the transcriptome and proteome diversity by altering transcription complexity in response to 


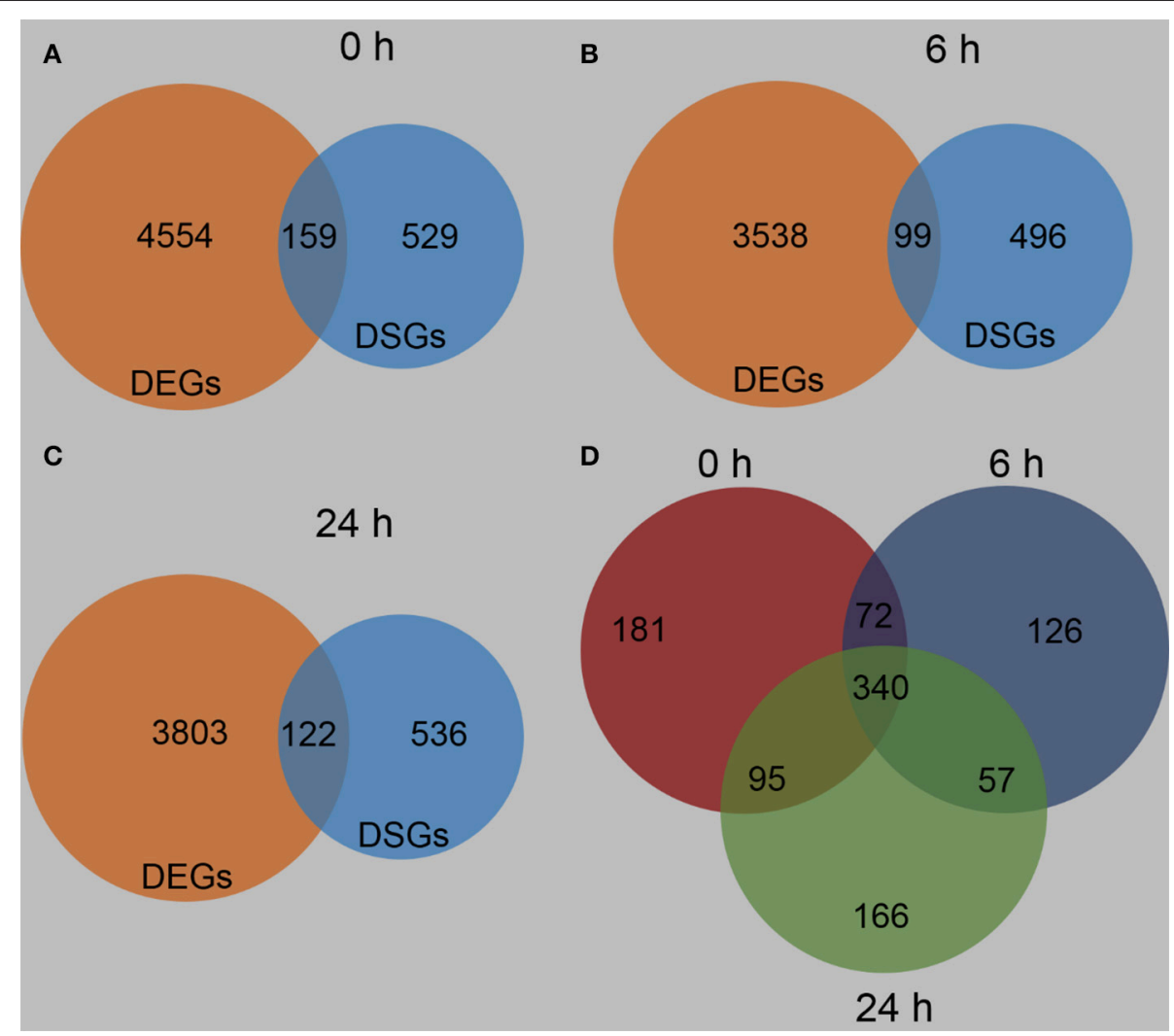

FIGURE 10 | Differentially spliced genes between B73 and Mo17. The fourth maize leaves were each treated with 50 aphids, and samples were collected at 6 and $24 \mathrm{~h}$ (untreated leaves served as controls, depicted as $0 \mathrm{~h}$ ). Venn diagrams indicating the differentially expressed genes (DEGs) and differentially spliced genes (DSGs) between B73 and Mo17 at $0 \mathrm{~h}$ (A), $6 \mathrm{~h}$ (B), and $24 \mathrm{~h}$ (C). Venn diagram indicating specific and overlapping DSGs between B73 and Mo17 at 0, 6 , and $24 \mathrm{~h}$ of $R$. padi feeding (D). Full descriptions can be found in Tables S6, S10.

stresses (Gassmann, 2008; Syed et al., 2012; Reddy et al., 2013; Yang et al., 2014). Our comparison of DEGs and DSGs between B73 and Mo17 under control, 6 and $24 \mathrm{~h}$ of aphid herbivory indicated that only very few genes were regulated on both expression and AS level (Figures 10A-C, Tables S6, S10). It is likely that AS and gene expression are regulated largely independent in maize response to aphid feeding. This is consistent with a study on AS events in the response of $N$. attenuata to the chewing caterpillar M. sexta, where $5 \mathrm{~h}$ of $M$. sexta feeding induced 180 and 356 DSGs in leaves and roots, respectively, and there was only little overlap (31 genes in leaves and six genes in roots) between differentially expressed and DSGs in both leaves and roots (Ling et al., 2015). After R. padi feeding, B73 and Mo17 had only some genes that were DSGs at both 6 and $24 \mathrm{~h}$ (Figures 9C,F, Table S9), indicating a highly time-specific regulation of AS. The finding that B73 and Mo17 have many specific DSGs under control and $R$. padi-feeding conditions (Figure 10D, Table S10) also suggest an important role of AS in diversifying the specific responses to R. padi infestation on different maize lines. A growing body of evidence has revealed that AS influences gene functions in plants. For example, the FLK protein influences flowering time in Arabidopsis, and FLK in prmt5 mutants (impaired in
AS regulation) cannot be normally spliced, resulting in a late flowering phenotype (Pei et al., 2007; Deng et al., 2010); PRR9 is a core-clock gene, which influences the circadian rhythm in Arabidopsis, and altered splicing of PRR9 caused an impairment of the circadian clock (Sanchez et al., 2010); JAZ proteins play an important role in repressing jasmonic acid signaling. AS of JAZ10 produces different splice variants, and JA-induced JAZ10.4 protein accumulation has been suggested to be an important feedback regulator to mediate JA signal desensitization (Moreno et al., 2013). Further functional studies on the different spliced isoforms of alternatively spliced genes are needed to understand if and how AS regulates maize responses to aphid herbivory.

Taken together, our large-scale data analyses revealed highly distinct metabolic and transcriptomic features in B73 and Mo17, and the differences remained large after aphid R. padi feeding (Figure 11). Furthermore, we identified a group of TFs that might contribute to the different resistance to $R$. padi by regulating the accumulation of Bxs. These datasets could be used for further dissecting the genetic basis for maize defense against aphids and for breeding new maize varieties with enhanced aphid resistance. 


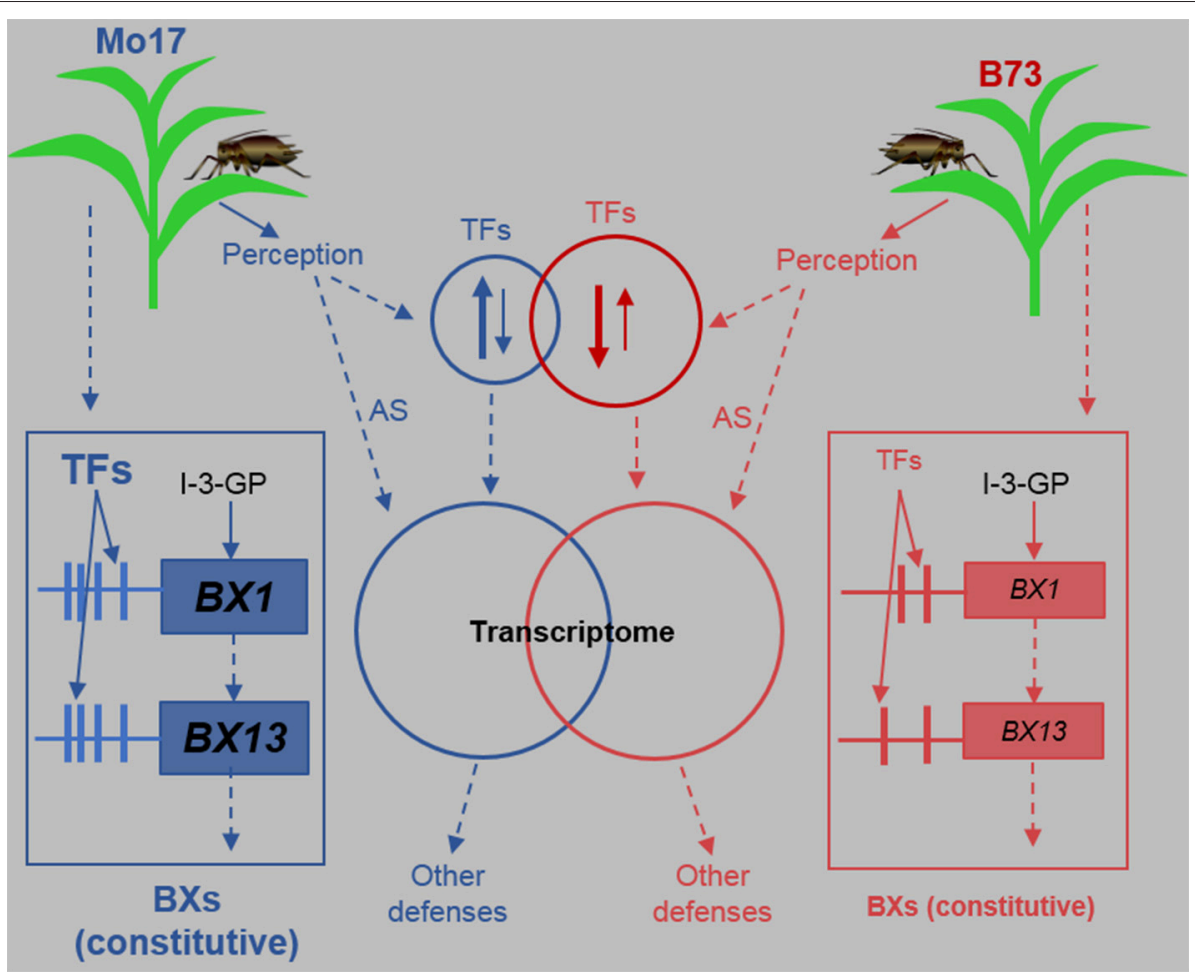

FIGURE 11 | A model summarizing the defense responses of B73 and Mo17 against the aphid $R$. padi. Maize plants respond to $R$. padi feeding mainly by perceiving certain aphid-derived elicitors through unknown receptors, thereby transcriptionally up- or down-regulating TFs (transcription factors). In B73, more TFs are regulated than in Mo17 and most TFs are down-regulated, while in Mo17 most TFs are up-regulated. TFs and other pathways, including alternative splicing (AS), continuously shape the transcriptomes of B73 and Mo17, resulting in changes in defenses. Importantly, certain TFs, that are not regulated by R. padi herbivory, target the promoters of $B X 1$ and $B X 13$ and have higher activity in Mo17 than in B73, resulting in much higher expression levels of $B \times 1$ and $B \times 13$ and thus high contents of benzoxazinoids (BXs) in Mo17. The promoters of BX1 and BX13 in Mo17 may also possess more cis-elements that allow more efficient transcription. I-3-GP: indole-3-glycerol phosphate; Red and blue arrows and circles represent B73 and Mo17, respectively. Up and down arrows in the circles indicate up-regulation or down-regulation, and the thickness of these arrows represent the numbers of genes. The sizes of the circles indicate the number of regulated TF genes and the size of letters represent activity, expression, or concentrations. Vertical lines connected with the boxes of $B \times 1$ and $B \times 13$ represent their promoters, and the horizontal bars symbolize cis-elements. Bxs are a group of metabolites produced by BX1 to BX14.

\section{AUTHOR CONTRIBUTIONS}

JW conceived and designed the study. JQ, JL, and $\mathrm{CH}$ provided advice on the experimental design. SW and JS performed phenotype measurements. JS, HL, HZ, CZ, and YX analyzed data. JS and JW wrote the manuscript. All authors reviewed and edited the manuscript.

\section{FUNDING}

This work was supported by the National Natural Science Foundation of China (Nos. U1502263 and 31401757), the Strategic Priority Research Program of the Chinese Academy of

\section{REFERENCES}

Ahmad, S., Veyrat, N., Gordon-Weeks, R., Zhang, Y., Martin, J., Smart, L., et al. (2011). Benzoxazinoid metabolites regulate innate immunity against aphids and fungi in maize. Plant Physiol. 157, 317-327. doi: 10.1104/pp.111. 180224
Sciences (No. XDB11050200), and the Max Planck Partner Group program.

\section{ACKNOWLEDGMENTS}

We thank Dr. Matthias Erb (University of Bern) for providing Bx standards.

\section{SUPPLEMENTARY MATERIAL}

The Supplementary Material for this article can be found online at: https://www.frontiersin.org/articles/10.3389/fpls.2017. 01738/full\#supplementary-material

Alborn, H. T., Turlings, T. C. J., Jones, T. H., Stenhagen, G., Loughrin, J. H., and Tumlinson, J. H. (1997). An elicitor of plant volatiles from beet armyworm oral secretion. Science 276, 945-949. doi: 10.1126/science.276.5314.945

Appel, H. M., Fescemyer, H., Ehlting, J., Weston, D., Rehrig, E., Joshi, T., et al. (2014). Transcriptional responses of Arabidopsis thaliana to chewing and sucking insect herbivores. Front. Plant Sci. 5:565. doi: 10.3389/fpls.2014.00565 
Argandona, V. H., Corcuera, L. J., Niemeyer, H. M., and Campbell, B. C. (1983). Toxicity and feeding deterrency of hydroxamic acids from gramineae in synthetic diets against the greenbug, Schizaphis graminum. Entomol. Exp. Appl. 34, 134-138. doi: 10.1111/j.1570-7458.1983.tb03307.x

Betsiashvili, M., Ahern, K. R., and Jander, G. (2015). Additive effects of two quantitative trait loci that confer Rhopalosiphum maidis (corn leaf aphid) resistance in maize inbred line Mo17. J. Exp. Bot. 66, 571-578. doi: $10.1093 / \mathrm{jxb} / \mathrm{eru} 379$

Black, D. L. (2003). Mechanisms of alternative pre-messenger RNA splicing. Annu. Rev. Biochem. 72, 291-336. doi: 10.1146/annurev.biochem.72.121801.161720

Bohlmann, J., Meyer-Gauen, G., and Croteau, R. (1998). Plant terpenoid synthases: Molecular biology and phylogenetic analysis. Proc. Natl. Acad. Sci. U.S.A. 95, 4126-4133. doi: 10.1073/pnas.95.8.4126

Buckler, E. S., Gaut, B. S., and McMullen, M. D. (2006). Molecular and functional diversity of maize. Curr. Opin. Plant Biol. 9, 172-176. doi: 10.1016/j.pbi.2006.01.013

Butrón, A., Chen, Y. C., Rottinghaus, G. E., and McMullen, M. D. (2010). Genetic variation at bx1 controls DIMBOA content in maize. Theor. Appl. Genet. 120, 721-734. doi: 10.1007/s00122-009-1192-1

Carena, M. J., and Glogoza, P. (2004). Resistance of maize to the corn leaf aphid: A review. Maydica 49, 241-254.

Cheng, A. X., Lou, Y. G., Mao, Y. B., Lu, S., Wang, L. J., and Chen, X. Y. (2007). Plant terpenoids: biosynthesis and ecological functions. J. Integr. Plant Biol. 49, 179-186. doi: 10.1111/j.1744-7909.2007.00395.x

Cooper, W. R., and Goggin, F. L. (2005). Effects of jasmonate-induced defenses in tomato on the potato aphid, Macrosiphum euphorbiae. Entomol. Exp. Appl. 115, 107-115. doi: 10.1111/j.1570-7458.2005.00289.x

de Vos, M., and Jander, G. (2010). Volatile communication in plant-aphid interactions. Curr. Opin. Plant Biol. 13, 366-371. doi: 10.1016/j.pbi.2010.05.001

De Vos, M., Van Oosten, V. R., Van Poecke, R. M., Van Pelt, J. A. V., Pozo, M. J., Mueller, M. J., et al. (2005). Signal signature and transcriptome changes of Arabidopsis during pathogen and insect attack. Mol. Plant Microbe 18, 923-937. doi: 10.1094/MPMI-18-0923

Deng, X., Gu, L., Liu, C., Lu, T., Lu, F., Lu, Z., et al. (2010). Arginine methylation mediated by the Arabidopsis homolog of PRMT5 is essential for proper pre-mRNA splicing. Proc. Natl. Acad. Sci. U.S.A. 107, 19114-19119. doi: 10.1073/pnas.1009669107

Du, Z., Zhou, X., Ling, Y., Zhang, Z., and Su, Z. (2010). agriGO: a GO analysis toolkit for the agricultural community. Nucleic Acids Res. 38, W64-W70. doi: $10.1093 / \mathrm{nar} / \mathrm{gkq} 310$

Dudareva, N., Klempien, A., Muhlemann, J. K., and Kaplan, I. (2013). Biosynthesis, function and metabolic engineering of plant volatile organic compounds. New Phytol. 198, 16-32. doi: 10.1111/nph.12145

FAO (2017). The future of food and agriculture - Trends and challenges, Rome: FAO.

Gassmann, W. (2008). Alternative splicing in plant defense. Curr. Top. Microbiol. Immunol. 326, 219-233. doi: 10.1007/978-3-540-76776-3_12

Glauser, G., Marti, G., Villard, N., Doyen, G. A., Wolfender, J. L., Turlings, T. C., et al. (2011). Induction and detoxification of maize 1,4-benzoxazin-3-ones by insect herbivores. Plant J. 68, 901-911. doi: 10.1111/j.1365-313X.2011.04740.x

Goggin, F. L. (2007). Plant-aphid interactions: molecular and ecological perspectives. Curr. Opin. Plant Biol. 10, 399-408. doi: 10.1016/j.pbi.2007.06.004

Guerrieri, E., and Digilio, M. C. (2008). Aphid-plant interactions: a review. J. Plant Interact. 3, 223-232. doi: 10.1080/17429140802567173

Hales, D. F., Tomiuk, J., Wohrmann, K., and Sunnucks, P. (1997). Evolutionary and genetic aspects of aphid biology: a review. Eur. J. Entomol. 94, 1-55.

Handrick, V., Robert, C. A., Ahern, K. R., Zhou, S., Machado, R. A., Maag, D., et al. (2016). Biosynthesis of 8-O-methylated benzoxazinoid defense compounds in Maize. Plant Cell 28, 1682-1700. doi: 10.1105/tpc.16.00065

Heil, M. (2008). Indirect defence via tritrophic interactions. New Phytol. 178, 41-61. doi: 10.1111/j.1469-8137.2007.02330.x

Hu, Q. P., and Xu, J. G. (2011). Profiles of carotenoids, anthocyanins, phenolics, and antioxidant activity of selected color waxy corn grains during maturation. J. Agr. Food Chem. 59, 2026-2033. doi: 10.1021/jf104149q

Huggett, D. A. J., Leather, S. R., and Walters, K. F. A. (1999). Suitability of the biomass crop Miscanthus sinensis as a host for the aphids Rhopalosiphum padi (L.) and Rhopalosiphum maidis (F.), and its susceptibility to the plant luteovirus Barley Yellow Dwarf Virus. Agr. Forest Entomol. 1, 143-149. doi: 10.1046/j.1461-9563.1999.00019.x

Jiang, J., Liu, X., Liu, C., Liu, G., Li, S., and Wang, L. (2017). Integrating omics and alternative splicing reveals insights into grape response to high temperature. Plant Physiol. 173, 1502-1518. doi: 10.1104/pp.16.01305

Jiao, Y., Zhao, H., Ren, L., Song, W., Zeng, B., Guo, J., et al. (2012). Genome-wide genetic changes during modern breeding of maize. Nat. Genet. 44, 812-815. doi: $10.1038 /$ ng.2312

Jin, J., He, K., Tang, X., Li, Z., Lv, L., Zhao, Y., et al. (2015). An Arabidopsis transcriptional regulatory map reveals distinct functional and evolutionary features of novel transcription factors. Mol. Biol. Evol. 32, 1767-1773. doi: 10.1093/molbev/msv058

Jin, J., Tian, F., Yang, D. C., Meng, Y. Q., Kong, L., Luo, J., et al. (2017). PlantTFDB 4.0: toward a central hub for transcription factors and regulatory interactions in plants. Nucleic Acids Res. 45, D1040-D1045. doi: 10.1093/nar/gkw982

Joung, J. G., Corbett, A. M., Fellman, S. M., Tieman, D. M., Klee, H. J., Giovannoni, J. J., et al. (2009). Plant MetGenMAP: an integrative analysis system for plant systems biology. Plant Physiol. 151, 1758-1768. doi: 10.1104/pp.109.145169

Keller, M., Hu, Y., Mesihovic, A., Fragkostefanakis, S., Schleiff, E., and Simm, S. (2016). Alternative splicing in tomato pollen in response to heat stress. DNA Res. 24, 205-217. doi: 10.1093/dnares/dsw051

Kettles, G. J., Drurey, C., Schoonbeek, H. J., Maule, A. J., and Hogenhout, S. A. (2013). Resistance of Arabidopsis thaliana to the green peach aphid, Myzus persicae, involves camalexin and is regulated by microRNAs. New Phytol. 198, 1178-1190. doi: $10.1111 /$ nph. 12218

Kim, D., Pertea, G., Trapnell, C., Pimentel, H., Kelley, R., and Salzberg, S. L. (2013). TopHat2: accurate alignment of transcriptomes in the presence of insertions, deletions and gene fusions. Genome Biol. 14:R36. doi: 10.1186/gb-2013-14-4-r36

Leszczynski, B., Warchol, J., and Niraz, S. (1985). The influence of phenolic compounds on the preference of winter wheat cultivars by cereal aphids. Insect. Sci. Appl. 6, 157-158.

Ling, Z., Zhou, W., Baldwin, I. T., and Xu, S. (2015). Insect herbivory elicits genome-wide alternative splicing responses in Nicotiana attenuata. Plant J. 84, 228-243. doi: 10.1111/tpj.12997

Long, B. J., Dunn, G. M., Bowman, J. S., and Routley, D. G. (1977). Relationship of hydroxamic acid content in corn and resistance to corn leaf aphid. Crop Sci. 17, 55-58. doi: 10.2135/cropsci1977.0011183X001700010016x

Mao, J., and Zeng, F. (2014). Plant-mediated RNAi of a gap gene-enhanced tobacco tolerance against the Myzus persicae. Transgenic Res. 23, 145-152. doi: 10.1007/s11248-013-9739-y

Meihls, L. N., Handrick, V., Glauser, G., Barbier, H., Kaur, H., Haribal, M. M., et al. (2013). Natural variation in maize aphid resistance is associated with 2,4-Dihydroxy-7-Methoxy-1,4-Benzoxazin-3-One glucoside methyltransferase activity. Plant Cell 25, 2341-2355. doi: 10.1105/tpc.113.112409

Meihls, L. N., Kaur, H., and Jander, G. (2012). Natural variation in maize defense against insect herbivores. Cold Spring Harb. Symp. Quant. Biol. 77, 269-283. doi: 10.1101/sqb.2012.77.014662

Moreno, J. E., Shyu, C., Campos, M. L., Patel, L. C., Chung, H. S., Yao, J., et al. (2013). Negative feedback control of jasmonate signaling by an alternative splice variant of JAZ10. Plant Physiol. 162, 1006-1017. doi: 10.1104/pp.113.218164

Niemeyer, H. M. (2009). Hydroxamic acids derived from 2-Hydroxy-2H-1,4Benzoxazin-3(4H)-one: key defense chemicals of cereals. J. Agr. Food Chem. 57, 1677-1696. doi: 10.1021/jf8034034

Oerke, E. C. (2005). Crop losses to pests. J. Agric. Sci. 144, 31-43. doi: 10.1017/S0021859605005708

Pei, Y. X., Niu, L. F., Lu, F. L., Liu, C. Y., Zhai, J. X., Kong, X. F., et al. (2007). Mutations in the type II protein arginine methyltransferase AtPRMT5 result in pleiotropic developmental defects in Arabidopsis(1[C][OA]). Plant Physiol. 144, 1913-1923. doi: 10.1104/pp.107.099531

Qi, J., Sun, G., Wang, L., Zhao, C., Hettenhausen, C., Schuman, M. C., et al. (2016). Oral secretions from Mythimna separata insects specifically induce defense responses in maize as revealed by high-dimensional biological data. Plant Cell Environ. 39, 1749-1766. doi: 10.1111/pce.12735

Razmjou, J., and Golizadeh, A. (2010). Performance of corn leaf aphid, Rhopalosiphum maidis (Fitch) (Homoptera: Aphididae) on selected maize 
hybrids under laboratory conditions. Appl. Entomol. Zool. 45, 267-274. doi: 10.1303/aez.2010.267

Reddy, A. S., Marquez, Y., Kalyna, M., and Barta, A. (2013). Complexity of the alternative splicing landscape in plants. Plant Cell 25, 3657-3683. doi: $10.1105 /$ tpc. 113.117523

Ren, Z. J., Liu, Y., Kang, D., Fan, K. J., Wang, C. Y., Wang, G. Y., et al. (2015). Two alternative splicing variants of maize HKT1;1 confer salt tolerance in transgenic tobacco plants. Plant Cell Tiss. Org. 123, 569-578. doi: 10.1007/s11240-015-0861-9

Romanowski, A., and Yanovsky, M. J. (2015). Circadian rhythms and post-transcriptional regulation in higher plants. Front. Plant Sci. 6:437. doi: $10.3389 /$ fpls.2015.00437

Rostas, M. (2007). The effects of 2,4-dihydroxy-7-methoxy-1,4-benzoxazin-3-one on two species of Spodoptera and the growth of Setosphaeria turcica in vitro. J. Pest Sci. 80, 35-41. doi: 10.1007/s10340-006-0151-8

Saeed, A. I., Sharov, V., White, J., Li, J., Liang, W., Bhagabati, N., et al. (2003). TM4: A free, open-source system for microarray data management and analysis. BioTechniques 34, 374.

Sammeth, M., Foissac, S., and Guigó, R. (2008). A general definition and nomenclature for alternative splicing events. PLoS Comput. Biol. 4:e1000147. doi: 10.1371/journal.pcbi.1000147

Sanchez, S. E., Petrillo, E., Beckwith, E. J., Zhang, X., Rugnone, M. L., Hernando, C. E., et al. (2010). A methyl transferase links the circadian clock to the regulation of alternative splicing. Nature 468, 112-116. doi: 10.1038/nature09470

Schliebner, I., Becher, R., Hempel, M., Deising, H. B., and Horbach, R. (2014). New gene models and alternative splicing in the maize pathogen Colletotrichum graminicola revealed by RNA-Seq analysis. BMC Genomics 15:842. doi: 10.1186/1471-2164-15-842

Schnee, C., Köllner, T. G., Gershenzon, J., and Degenhardt, J. (2002). The maize gene terpene synthase 1 encodes a sesquiterpene synthase catalyzing the formation of (E)-beta-farnesene, (E)-nerolidol, and (E,E)-farnesol after herbivore damage. Plant Physiol. 130, 2049-2060. doi: 10.1104/pp.008326

Schnee, C., Köllner, T. G., Held, M., Turlings, T. C., Gershenzon, J., and Degenhardt, J. (2006). The products of a single maize sesquiterpene synthase form a volatile defense signal that attracts natural enemies of maize herbivores. Proc. Natl. Acad. Sci. U.S.A. 103, 1129-1134. doi: 10.1073/pnas. 0508027103

Seo, P. J., Park, M. J., and Park, C. M. (2013). Alternative splicing of transcription factors in plant responses to low temperature stress: mechanisms and functions. Planta 237, 1415-1424. doi: 10.1007/s00425-013-1882-4

Simpson, C. G., Lewandowska, D., Fuller, J., Maronova, M., Kalyna, M., Davidson, D., et al. (2008). Alternative splicing in plants. Biochem. Soc. 36, 508-510. doi: $10.1042 /$ BST0360508

Smith, C. M., and Boyko, E. V. (2007). The molecular bases of plant resistance and defense responses to aphid feeding: current status. Entomol. Exp. Appl. 122, 1-16. doi: 10.1111/j.1570-7458.2006.00503.x

Springer, N. M., Ying, K., Fu, Y., Ji, T., Yeh, C. T., Jia, Y., et al. (2009). Maize inbreds exhibit high levels of Copy Number Variation (CNV) and Presence/Absence Variation (PAV) in genome content. PLoS Genet. 5:734. doi: 10.1371/journal.pgen.1000734

Srinivasan, A., Jiménez-Gómez, J. M., Fornara, F., Soppe, W. J., and Brambilla, V. (2016). Alternative splicing enhances transcriptome complexity in desiccating seeds. J. Integr. Plant Biol. 58, 947-958. doi: 10.1111/jipb.12482

Syed, N. H., Kalyna, M., Marquez, Y., Barta, A., and Brown, J. W. (2012). Alternative splicing in plants - coming of age. Trends Plant Sci. 17, 616-623. doi: 10.1016/j.tplants.2012.06.001

Tamiru, A., Bruce, T. J. A., Richter, A., Woodcock, C. M., Midega, C. A. O., Degenhardt, J., et al. (2017). A maize landrace that emits defense volatiles in response toherbivore eggs possesses a strongly inducible terpene synthase gene. Ecol. Evol. 7, 2835-2845. doi: 10.1002/ece3.2893

Tamiru, A., Bruce, T. J., Woodcock, C. M., Caulfield, J. C., Midega, C. A., Ogol, C. K, et al. (2011). Maize landraces recruit egg and larval parasitoids in response to egg deposition by a herbivore. Ecol. Lett. 14, 1075-1083. doi: 10.1111/j.1461-0248.2011.01674.x
Thackray, D. J., Wratten, S. D., Edwards, P. J., and Niemeyer, H. M. (1990). Resistance to the Aphids Sitobion avenae and Rhopalosiphum padi in gramineae in relation to hydroxamic acid levels. Ann. Appl. Biol. 116, 573-582. doi: 10.1111/j.1744-7348.1990.tb06640.x

Thatcher, S. R., Danilevskaya, O. N., Meng, X., Beatty, M., Zastrow-Hayes, G., Harris, C., et al. (2016). Genome-wide analysis of alternative splicing during development and drought stress in maize. Plant Physiol. 170, 586-599. doi: $10.1104 /$ pp. 15.01267

Thompson, G. A., and Goggin, F. L. (2006). Transcriptomics and functional genomics of plant defence induction by phloem-feeding insects. J. Exp. Bot. 57, 755-766. doi: 10.1093/jxb/erj135

Trapnell, C., Roberts, A., Goff, L., Pertea, G., Kim, D., Kelley, D. R., et al. (2012). Differential gene and transcript expression analysis of RNAseq experiments with TopHat and Cufflinks. Nat. Protoc. 7, 562-578. doi: 10.1038/nprot.2012.016

Tzin, V., Fernandez-Pozo, N., Richter, A., Schmelz, E. A., Schoettner, M., Schäfer, M., et al. (2015a). Dynamic maize responses to aphid feeding are revealed by a time series of transcriptomic and metabolomic assays. Plant Physiol. 169, 1727-1743. doi: 10.1104/pp.15.01039

Tzin, V., Lindsay, P. L., Christensen, S. A., Meihls, L. N., Blue, L. B., and Jander, G. (2015b). Genetic mapping shows intraspecific variation and transgressive segregation for caterpillar-induced aphid resistance in maize. Mol. Ecol. 24, 5739-5750. doi: $10.1111 / \mathrm{mec} .13418$

Wahlroos, O., and Virtanen, A. (1959). Precursors of 6-methoxybenzoxazolinone in maize and wheat plants, their isolation and some of their properties. Acta Chem. Scand. 13, 1906-1908. doi: 10.3891/acta.chem.scand.13-1906

Waisi, H., Kosovic, A., Krstic, D., Milojkovic-Opsenica, D., Nikolic, B., Dragilevic, V., et al. (2015). Polyphenolic profile of maize seedlings treated with 24 -epibrassinolide. J. Chem. 2015, 1-10. doi: 10.1155/2015/976971

Wang, K., Jiao, Z., Xu, M., Wang, Y., Li, R., Cui, X., et al. (2017). Landscape and fruit developmental regulation of alternative splicing in tomato by genomewide analysis. Hortic. Plant J. 2, 338-350. doi: 10.1016/j.hpj.2017.01.007

Wang, Y., Wang, H., Fan, R., Yang, Q., and Yu, D. (2014). Transcriptome analysis of soybean lines reveals transcript diversity and genes involved in the response to common cutworm (Spodoptera litura Fabricius) feeding. Plant Cell Environ. 37, 2086-2101. doi: 10.1111/pce.12296

Wójcicka, A. (2010). Cereal phenolic compounds as biopesticides of cereal aphids. Polish. J. Environ. Stud. 19, 1337-1343.

$\mathrm{Wu}$, J., Hettenhausen, C., Schuman, M. C., and Baldwin, I. T. (2008). A comparison of two Nicotiana attenuata accessions reveals large differences in signaling induced by oral secretions of the specialist herbivore Manduca sexta. Plant Physiol. 146, 927-939. doi: 10.1104/pp.107.114785

Yang, S., Tang, F., and Zhu, H. (2014). Alternative splicing in plant immunity. Int. J. Mol. Sci. 15, 10424-10445. doi: 10.3390/ijms150610424

Ye, J., Fang, L., Zheng, H., Zhang, Y., Chen, J., Zhang, Z., et al. (2006). WEGO: a web tool for plotting GO annotations. Nucleic Acids Res. 34, W293-W297. doi: 10.1093/nar/gkl031

Zheng, L., McMullen, M. D., Bauer, E., Schön, C. C., Gierl, A., and Frey, M. (2015). Prolonged expression of the BX1 signature enzyme is associated with a recombination hotspot in the benzoxazinoid gene cluster in Zea mays. J. Exp. Bot. 66, 3917-3930. doi: 10.1093/jxb/erv192

Conflict of Interest Statement: The authors declare that the research was conducted in the absence of any commercial or financial relationships that could be construed as a potential conflict of interest.

Copyright (c) 2017 Song, Liu, Zhuang, Zhao, Xu, Wu, Qi, Li, Hettenhausen and Wu. This is an open-access article distributed under the terms of the Creative Commons Attribution License (CC BY). The use, distribution or reproduction in other forums is permitted, provided the original author(s) or licensor are credited and that the original publication in this journal is cited, in accordance with accepted academic practice. No use, distribution or reproduction is permitted which does not comply with these terms. 